\title{
Control of Systems With Slow Actuators Using Time Scale Separation
}

\author{
Vahram Stepanyan* \\ Mission Critical Technologies Inc, NASA Ames Research Center, Moffett Field, CA 94035, \\ Nhan Nguyen ${ }^{\dagger}$ \\ NASA Ames Research Center, Moffett Field, CA 94043
}

\begin{abstract}
This paper addresses the problem of controlling a nonlinear plant with a slow actuator using singular perturbation method. For the known plant-actuator cascaded system the proposed scheme achieves tracking of a given reference model with considerably less control demand than would otherwise result when using conventional design techniques. This is the consequence of excluding the small parameter from the actuator dynamics via time scale separation. The resulting tracking error is within the order of this small parameter. For the unknown system the adaptive counterpart is developed based on the prediction model, which is driven towards the reference model by the control design. It is proven that the prediction model tracks the reference model with an error proportional to the small parameter, while the prediction error converges to zero. The resulting closed-loop system with all prediction models and adaptive laws remains stable. The benefits of the approach are demonstrated in simulation studies and compared to conventional control approaches.
\end{abstract}

\section{Introduction}

In many applications, the plant to be controlled has much slower dynamics than the actuator through which it is being controlled. That is, the cascaded plant-actuator system can be described as

$$
\begin{aligned}
\dot{\boldsymbol{x}}(t) & =\boldsymbol{f}(\boldsymbol{x}(t))+\boldsymbol{g}(x(t)) y(t) \\
\varepsilon \dot{\boldsymbol{z}}(t) & =\boldsymbol{a}(z(t))+\boldsymbol{b}(z(t)) u(t) \\
y(t) & =c(z(t)),
\end{aligned}
$$

where $x(t) \in \mathbb{R}^{n}$ is the plant's state, $\boldsymbol{z}(t) \in \mathbb{R}^{m}$ is the actuator's state, $y(t) \in \mathbb{R}$ is the actuator's output, $u(t) \in \mathbb{R}$ is the actuator's input, and $\varepsilon$ is a small constant. For these types of systems, the singular perturbations method has been successfully used for control design. ${ }^{6}$ This approach requires some interconnection conditions to be satisfied, which can be imposed directly on the systems under the consideration (See for example ${ }^{16}$ ) or in terms of Lyapunov functions and their derivatives (See for example ${ }^{13}$ ). The developed controllers typically achieve local results (stability or asymptotic stability) of closed-loop systems (See for example Refs. ${ }^{2,6,8}$ just to mention a few of them). However, for some systems, global exponential stability can be achieved. ${ }^{1}$ As it is shown in Ref., ${ }^{5}$ when the exponential stability is achieved, the actuator's dynamics can be neglected and the actuator's output can be viewed as a control input for the plant. Therefore, the control design can follow any known scheme that is suitable for the given plant dynamics.

In some applications, the actuator dynamics can be substantially slower than the plant's dynamics. That is, the cascaded plant-actuator system is now described by the equations

$$
\begin{aligned}
\dot{\boldsymbol{x}}(t) & =\boldsymbol{f}(\boldsymbol{x}(t))+\boldsymbol{g}(x(t)) y(t) \\
\varepsilon^{-1} \dot{\boldsymbol{z}}(t) & =\boldsymbol{a}(z(t))+\boldsymbol{b}(z(t)) u(t) \\
y(t) & =c(z(t)) .
\end{aligned}
$$

\footnotetext{
*Senior Scientist, Mission Critical Technologies Inc., Senior Member AIAA, vahram.stepanyan@nasa.gov

${ }^{\dagger}$ Project Scientist, Integrated Resilient Aircraft Control Project, Intelligent Systems Division, Associate Fellow AIAA, nhan.t.nguyen@nasa.gov
} 
This is the case when the specific actuator, which is designed to control the plant in ordinary scenarios, fails, and the substitute is used to rescue the emergency situation. For example, the aircraft engine can be used for the directional stability and control of the aircraft when the control surface deflection system fails. However, the engine dynamics is known to be much slower than the aircraft's rotational dynamics with the rudder input. For these types of problems, the actuator dynamics cannot be ignored, and the actual control input has to be designed to generate the necessary actuator output. In general, the actual control input depends is inversely proportional to the small parameter, thus resulting in high gain control signal. Therefore, to generate the necessary torque or force, the actuator may require a control signal exceeding the physical limits.

The goal of this paper is to show that the application of the singular perturbation method based time scale separation can avoid demanding high magnitude control signals, and to develop a control algorithm that guarantees the stable tracking of a given reference command in the presence of modeling uncertainties and external disturbances.

The rest of the paper is organized as follows. First, we give preliminaries for the singular perturbations method, then we apply the method to the known plant's and actuator's dynamics. Afterwards, we present time scale separation based control design for the unknown system and provide stability analysis. Throughout the paper bold symbols are used for vectors, capital letters for matrices and small letters for scalars.

\section{Preliminaries}

Consider the singular perturbation model (1) with initial conditions $\boldsymbol{x}(0)=\boldsymbol{x}_{0}, \boldsymbol{z}(o)=\boldsymbol{z}_{0}$. We assume that the functions $\boldsymbol{f}(\boldsymbol{x})$ and $\boldsymbol{g}(\boldsymbol{x})$ are continuously differentiable on some open connected set $D_{x} \subset \mathbb{R}^{n}$, and the functions $\boldsymbol{a}(\boldsymbol{z})$ and $\boldsymbol{b}(\boldsymbol{z})$ are continuously differentiable on some open connected set $D_{z} \subset \mathbb{R}^{m}$. Let the control input have the form $u(t)=\varphi(t, \boldsymbol{x}, \boldsymbol{z})$, where the function $\varphi(t, \boldsymbol{x}, \boldsymbol{z})$ and its first partial derivatives with respect to $(t, \boldsymbol{x}, \boldsymbol{z})$ are continuous and bounded on any compact subset of $\times D_{x} \times D_{z}$.

The model is said to be in standard form if the algebraic equation

$$
\boldsymbol{a}(\boldsymbol{z})+\boldsymbol{b}(\boldsymbol{z}) \varphi(t, \boldsymbol{x}, \boldsymbol{z})=0
$$

has isolated roots for all $(t, \boldsymbol{x}) \in[0, \infty) \times D_{x}$.

Let $\boldsymbol{z}=\boldsymbol{h}(t, \boldsymbol{x})$ be an isolated root of (3). The system

$$
\dot{\boldsymbol{x}}(t)=\boldsymbol{f}(\boldsymbol{x}(t))+\boldsymbol{g}(\boldsymbol{x}(t)) \boldsymbol{h}(t, \boldsymbol{x}(t)), \quad \boldsymbol{x}(0)=\boldsymbol{x}_{0}
$$

is called a reduced system. Denote the solution of (4) by $\overline{\boldsymbol{x}}(t)$.

Introduce a change of variables $\boldsymbol{\xi}=\boldsymbol{z}-\boldsymbol{h}(t, \boldsymbol{x})$ and $t=\varepsilon \tau$. The system

$$
\frac{d \boldsymbol{\xi}(\tau)}{d \tau}=\boldsymbol{a}(\boldsymbol{\xi}+\boldsymbol{h}(t, \boldsymbol{x}))+\boldsymbol{b}(\boldsymbol{\xi}+\boldsymbol{h}(t, \boldsymbol{x})) \varphi(t, \boldsymbol{x}, \boldsymbol{\xi}+\boldsymbol{h}(t, \boldsymbol{x})), \quad \boldsymbol{\xi}(0)=\boldsymbol{z}_{0}-\boldsymbol{h}\left(0, \boldsymbol{x}_{0}\right)
$$

where $(t, \boldsymbol{x}) \in[0, \infty) \times D_{x}$ are treated as fixed parameters, is called a boundary layer system.

In this paper, for the stability analysis of the closed-loop systems, we will use the following version of Theorem 11.2 from Ref. ${ }^{5}$

Theorem 1 Consider the singular perturbation problem for system (1) with initial conditions $\boldsymbol{x}(0)=\boldsymbol{x}_{0}$, $\boldsymbol{z}(o)=\boldsymbol{z}_{0}$ and control input $u(t)=\varphi(t, \boldsymbol{x}, \boldsymbol{z})$. Let $\boldsymbol{z}=\boldsymbol{h}(t, \boldsymbol{x})$ be an isolated root of the algebraic equation (3). Assume that the following conditions are satisfied

- The functions $\boldsymbol{f}(\boldsymbol{x})$ and $\boldsymbol{g}(\boldsymbol{x})$ are continuously differentiable on $D_{x}$;

- The function $\boldsymbol{a}(\boldsymbol{z}), \boldsymbol{b}(\boldsymbol{z})$ and $c(\boldsymbol{z})$ are continuously differentiable on $D_{z}$;

- The function $\varphi(t, \boldsymbol{x}, \boldsymbol{z})$ ant its first partial derivatives with respect to its arguments are continuous and bounded on any compact subset of $D_{x} \times D_{z}$;

- The origin is an exponentially stable equilibrium point of the reduced system (4), $\mathcal{R}_{x}$ is the region of attraction;

- The origin is an exponentially stable equilibrium point of the boundary-layer system (5), uniformly in $(t, \boldsymbol{x}), \mathcal{R}_{z}$ is the region of attraction. 
Then, for each compact set $\Omega_{x} \subset \mathcal{R}_{x}$ and $\Omega_{z} \subset \mathcal{R}_{z}$ there is a positive constant $\varepsilon^{*}$ such that for all $t \geq 0$, $\boldsymbol{x}_{0} \in \Omega_{x}, \boldsymbol{z}_{0}-\boldsymbol{h}\left(0, \boldsymbol{x}_{0}\right) \in \Omega_{z}$ and $0<\varepsilon<\varepsilon^{*}$, the singular perturbation problem (1) has a unique solution $\boldsymbol{x}(t, \varepsilon), \boldsymbol{z}(t, \varepsilon)$ on $[0, \infty)$, and

$$
\boldsymbol{x}(t, \varepsilon)-\overline{\boldsymbol{x}}(t)=O(\varepsilon)
$$

holds uniformly for all $t \geq 0$. Moreover, there exists a time instance $T(\varepsilon)$ such that

$$
\boldsymbol{z}(t, \varepsilon)-\boldsymbol{h}(t, \overline{\boldsymbol{x}}(t))=O(\varepsilon)
$$

holds uniformly for all $t \geq T(\varepsilon)$.

\section{Problem Formulation}

Consider a single-input single-output system

$$
\begin{aligned}
\dot{\boldsymbol{x}}_{p}(t) & =A_{p} \boldsymbol{x}_{p}(t)+\boldsymbol{b}_{p}\left[f_{p}\left(\boldsymbol{x}_{p}(t)\right)+g_{p}\left(\boldsymbol{x}_{p}(t)\right) y_{a}(t)+d_{P}(t)\right] \\
y_{p}(t) & =\boldsymbol{c}_{p}^{\top} \boldsymbol{x}_{p}(t),
\end{aligned}
$$

with the initial conditions $\left.\boldsymbol{x}_{(} 0\right)=\boldsymbol{x}_{p 0}$, where $\boldsymbol{x}_{p} \in \mathbb{R}^{n}$ is the plant's state, $f_{p}\left(\boldsymbol{x}_{p}\right)$ and $g_{p}\left(\boldsymbol{x}_{p}\right)$ are continuously differentiable unknown functions representing the modeling uncertainties, and $y_{a}(t) \in \mathbb{R}$ is the control effort generated by a slow actuator. It is reasonable to assume that the actuator's dynamics are stable, minimum phase and of known relative degree $r$, and are described by the system

$$
\begin{aligned}
\varepsilon^{-1} \dot{\boldsymbol{x}}_{a}(t) & =A_{a} \boldsymbol{x}_{a}(t)+\boldsymbol{b}_{a}\left[\omega u(t)+f_{a}\left(y_{a}(t), \boldsymbol{p}_{a}\right)+d_{a}(t)\right] \\
y_{a}(t) & =\boldsymbol{c}_{a}^{\top} \boldsymbol{x}_{a}(t)
\end{aligned}
$$

with initial conditions $\boldsymbol{x}_{a}(0)=\boldsymbol{x}_{a 0}$. Here, $\boldsymbol{x}_{a} \in \mathbb{R}^{m}$ is the actuator's state, $A_{a}$ is a Hurwitz matrix, the transfer function $G_{a}(s)=\boldsymbol{c}_{a}^{\top}\left(s \mathbb{I}-A_{a}\right)^{-1} \boldsymbol{b}_{a}$ has stable zeros, $\varepsilon$ is a small parameter, $\omega \in(0,1]$ represents the possible reduction in actuator's control effectiveness, $f_{a}\left(y_{a}, \boldsymbol{p}_{a}\right)$ is a continuously differentiable function representing the modeling uncertainties, $u(t)$ is the actuator's control input, and time signals $d_{p}(t)$ and $d_{a}(t)$ represent unknown external disturbances.

We notice that the actuator's dynamics do not depend on the plant's state to be controlled. However, the dynamics may depend on other external variables, which are lumped into the parameter $\boldsymbol{p}_{a}$. In general, the actuator's state is not available for feedback. Therefore, the actuator related part of the overall control design must be considered in output feedback framework, assuming that the signal $y_{a}(t)$ is available for feedback.

To guarantee the controllability of the plant, we also assume that the pair $\left(A_{p}, \boldsymbol{b}_{p}\right)$ is controllable, the pair $\left(A_{p}, \boldsymbol{c}_{p}\right)$ is observable, and the function $g_{p}\left(\boldsymbol{x}_{p}\right)$ never crosses zero. Without loss of generality, we take $g_{p}\left(\boldsymbol{x}_{p}\right)$ as a positive function, that is, there exists a positive constant $g_{0}$ such that $g_{p}\left(\boldsymbol{x}_{p}\right) \geq g_{0}>0$.

The control objective is to design an input signal $u(t)$ for the actuator such that the system's output $y_{p}(t)$ tracks the output of a given reference model

$$
\begin{aligned}
\dot{\boldsymbol{x}}_{m}(t) & =A_{m} \boldsymbol{x}_{m}(t)+\boldsymbol{b}_{m}^{\top} y_{c}(t) \\
y_{m}(t) & =\boldsymbol{c}^{\top} \boldsymbol{x}_{m}(t),
\end{aligned}
$$

with initial conditions $\boldsymbol{x}_{m}(0)=\boldsymbol{x}_{m 0}$, where $y_{c}(t)$ is an external command that is bounded with its first time derivative. Here, $A_{m}$ is a Hurwitz matrix of the form $A_{m}=A_{p}-\boldsymbol{b}_{p} \boldsymbol{k}^{\top}$ for some control gain $\boldsymbol{k}$, and $\boldsymbol{b}_{m}=\nu \boldsymbol{b}_{p}$ with $\nu=\left(\boldsymbol{c}_{p}^{\top} A_{m}^{-1} \boldsymbol{b}_{p}\right)^{-1}$. Since $A_{m}$ is Hurwitz, there exist a symmetric positive definite matrix $P$ such that

$$
A_{m}^{\top} P+P A_{m}=-Q
$$

for some symmetric positive definite matrix $Q$. 


\section{Control design for known systems}

In this section, we show that the magnitude of a control signal designed by the proposed time scale separation based algorithm is smaller than the magnitude of a control signal generated by the conventional algorithms by a term that is of the order of $\varepsilon^{-1}$. For comparison, we use backstepping design, ${ }^{7}$ which is well suited for the cascaded system comprised of the dynamics in (8) and the actuator dynamics (9). To this end we assume that the systems under consideration are known and disturbance free. Also, for the purposes of this section we assume that the functions $\boldsymbol{f}_{p}\left(\boldsymbol{x}_{p}\right), \boldsymbol{g}_{p}\left(\boldsymbol{x}_{p}\right)$ and $g_{a}\left(y_{a}\right)$ are sufficiently smooth, and the reference command and its sufficiently many time derivatives are bounded. In this case, for convenience we write the actuator dynamics in the following normal form ${ }^{10}$

$$
\begin{aligned}
\varepsilon^{-1} \dot{\boldsymbol{x}}_{a}^{c}(t) & =A_{0} \boldsymbol{x}_{a}^{c}(t)+\boldsymbol{b}_{0}\left[-\boldsymbol{k}_{a}^{\top} \boldsymbol{x}_{a}(t)+v(t)\right] \\
\varepsilon^{-1} \dot{\boldsymbol{\xi}}_{a}^{c}(t) & =A_{0}^{c} \boldsymbol{\xi}_{a}^{c}(t)+\boldsymbol{b}_{a}^{c} \boldsymbol{x}_{a}^{c}(t) \\
y_{a}(t) & =\boldsymbol{c}_{0} \boldsymbol{x}_{a}^{c}(t),
\end{aligned}
$$

where $\boldsymbol{x}_{a}^{c} \in \mathbb{R}^{r}$ is the portion of the actuator's state corresponding to the chain of $r$ integrators represented by the triplet $\left(\boldsymbol{c}_{0}^{\top} \in \mathbb{R}^{r}, A_{0} \in \mathbb{R}^{r \times r}, \boldsymbol{b}_{0} \in \mathbb{R}^{r}\right)$ having the form

$$
A_{0}=\left[\begin{array}{cc}
0 & \mathbb{I}_{r-1} \\
0 & 0
\end{array}\right], \quad \boldsymbol{c}_{0}=\left[\begin{array}{c}
1 \\
\vdots \\
0
\end{array}\right], \quad \boldsymbol{b}_{0}=\left[\begin{array}{c}
0 \\
\vdots \\
1
\end{array}\right],
$$

$\boldsymbol{\xi}_{a}^{c} \in \mathbb{R}^{m-r}$ is the actuator's internal state, and the eigenvalues of $A_{0}^{c} \in \mathbb{R}^{(m-r) \times(m-r)}$ are the stable zeros of the actuator's transfer function $G_{a}(s)$. For the notational simplicity, we introduce a new control variable $v(t)=b \omega u(t)+b g_{a}\left(y_{a}(t), \boldsymbol{p}_{a}\right)$, where $b \neq 0$ is the high frequency gain. Since the function $g_{a}\left(\boldsymbol{x}_{a}, \boldsymbol{p}_{a}\right)$ and parameters $\omega$ and $b$ are assumed to be known, $u(t)$ can be readily computed after designing $v(t)$.

Let the tracking error be $\boldsymbol{e}(t)=\boldsymbol{x}_{p}(t)-\boldsymbol{x}_{m}(t)$. Its dynamics can be written as

$$
\dot{\boldsymbol{e}}(t)=A_{m} \boldsymbol{e}(t)+\boldsymbol{b}_{p}\left[\boldsymbol{k}^{\top} \boldsymbol{x}_{p}(t)+f_{p}\left(\boldsymbol{x}_{p}(t)\right)+g_{p}\left(\boldsymbol{x}_{p}(t)\right) y_{a}(t)-\nu y_{c}(t)\right] .
$$

Since for the error dynamics (13) there exists a continuously differentiable feedback control law in the form

$$
y_{a}(t)=\alpha_{1}\left(t, \boldsymbol{x}_{p}\right)=g_{p}^{-1}\left(\boldsymbol{x}_{p}(t)\right)\left[-\boldsymbol{k}^{\top} \boldsymbol{x}_{p}(t)-f_{p}\left(\boldsymbol{x}_{p}(t)\right)-\nu y_{c}(t)\right],
$$

that guarantees that the derivative of the radially unbounded Lyapunov function

$$
V_{1}(\boldsymbol{e}(t))=\boldsymbol{e}^{\top}(t) P \boldsymbol{e}(t)
$$

is negative definite for all $e \in \mathbb{R}^{n}$

$$
\dot{V}_{1}(t)=-e^{\top}(t) Q e(t)<0,
$$

the linear block backstepping lemma (Lemma 2.23) from Ref. ${ }^{7}$ can be applied. To be prepared for that application, we introduce change of variables $\boldsymbol{z}^{c}(t)=T^{c} \boldsymbol{x}_{a}^{c}(t)$, where $T^{c}=\operatorname{diag}\left(1, \varepsilon, \ldots, \varepsilon^{r-1}\right)$. In these new variables, the first equation in (12) takes the form

$$
\begin{aligned}
\dot{z}_{i}^{c}(t) & =z_{i+1}^{c}(t), \quad i=1, \ldots, r-1 \\
\varepsilon^{-r} \dot{z}_{r}^{0}(t) & =-\boldsymbol{k}_{a}^{\top} \boldsymbol{x}_{a}(t)+v(t) \\
y_{a}(t) & =z_{1}^{c}(t) .
\end{aligned}
$$

Denoting $\eta_{i}(t)=z_{i}^{c}(t)-\alpha_{i}(t, \boldsymbol{x}), i=1, \ldots, r$, where the stabilizing functions $\alpha_{i}\left(t, \boldsymbol{x}_{p}\right)$ are defined as

$$
\begin{aligned}
\alpha_{2}\left(t, \boldsymbol{x}_{p}\right) & =-\lambda_{1} \eta_{1}(t)+\dot{\alpha}_{1}\left(t, \boldsymbol{x}_{p}\right)-\boldsymbol{e}^{\top}(t) P \boldsymbol{b}_{p} g_{p}\left(\boldsymbol{x}_{p}(t)\right) \\
\alpha_{i}\left(t, \boldsymbol{x}_{p}\right) & =-\lambda_{i-1} \eta_{i-1}(t)-\eta_{i-2}(t)+\dot{\alpha}_{i-1}\left(t, \boldsymbol{x}_{p}\right), \quad i=3, \ldots, r
\end{aligned}
$$


and $\lambda_{i}>0, i=1, \ldots, r-1$ are design parameters, the error system is written in the form

$$
\begin{aligned}
& \underbrace{\left[\begin{array}{c}
\dot{\boldsymbol{e}}(t) \\
\dot{\eta}_{1}(t) \\
\vdots \\
\dot{\eta}_{r-1}(t)
\end{array}\right]}_{\dot{\boldsymbol{x}}(t)}=\underbrace{\left[\begin{array}{c}
A_{m} \boldsymbol{e}(t)+\boldsymbol{b}_{p} g_{p}\left(\boldsymbol{x}_{p}\right) \eta_{1}(t) \\
-\lambda_{1} \eta_{1}(t)+\eta_{2}(t)-\boldsymbol{e}^{\top}(t) P \boldsymbol{b}_{p} g_{p}\left(\boldsymbol{x}_{p}\right) \\
\vdots \\
-\eta_{r-2}(t)-\lambda_{r-1} \eta_{r-1}(t)
\end{array}\right]}_{\boldsymbol{f}(t, \boldsymbol{x})}+\underbrace{\left[\begin{array}{c}
0 \\
0 \\
\vdots \\
1
\end{array}\right]}_{\boldsymbol{g}} \eta_{r}(t) \\
& \varepsilon^{-r} \dot{\eta}_{r}(t)=-\boldsymbol{k}_{a}^{\top} \boldsymbol{x}_{a}(t)-\varepsilon^{-r} \dot{\alpha}_{r}\left(t, \boldsymbol{x}_{p}\right)+v(t)
\end{aligned}
$$

We define the control law as

$$
v_{b}(t)=k_{a}^{\top} \boldsymbol{x}_{a}(t)-\lambda_{r} \eta_{r}(t)-\varepsilon^{-r} \eta_{r-1}(t)+\varepsilon^{-r} \dot{\alpha}_{r}\left(t, \boldsymbol{x}_{p}\right)
$$

where $\lambda_{r}>0$ is a design parameter. Then the closed-loop error system is reduced to

$$
\begin{aligned}
\dot{\boldsymbol{x}}(t) & =\boldsymbol{f}(t, \boldsymbol{x}(t))+\boldsymbol{g} \eta_{r}(t) \\
\varepsilon^{-r} \dot{\eta}_{r}(t) & =-\lambda_{r} \eta_{r}(t)-\varepsilon^{-r} \eta_{r-1}(t) .
\end{aligned}
$$

Lemma 1 The control signal (20) guarantees the boundedness of all closed-loop signals and the exponential convergence of the errors $\boldsymbol{e}(t)$ and $\eta_{i}(t), i=1, \ldots, r$ to zero.

Proof. Consider the following candidate Lyapunov function

$$
V_{2}(\boldsymbol{e}(t), \eta(t))=V_{1}(\boldsymbol{e}(t))+\eta_{1}^{2}(t)+\cdots+\eta_{r}^{2}(t) .
$$

Its derivative can be readily computed to satisfy the inequality

$$
\dot{V}_{2}(t)=-\boldsymbol{e}^{\top}(t) Q \boldsymbol{e}(t)-\lambda_{1} \eta_{1}^{2}(t)-\cdots-\lambda_{r-1} \eta_{r-1}^{2}(t)-\varepsilon \lambda_{r} \eta_{r}^{2}(t)<-\mu V_{2}(t)
$$

for all $\boldsymbol{e}(t) \in \mathbb{R}^{n}$ and $z(t) \in \mathbb{R}$, where $\mu=\lambda_{\max }^{-1}(P) \min \left[\lambda_{\min }^{-1}(Q), \lambda_{1}, \ldots, \lambda_{r-1},-\varepsilon^{r} \lambda_{r}\right]$ with $\lambda_{\max }(P)$ denoting the maximum eigenvalue of the matrix $P$. Since $V_{2}(t)$ is radially unbounded, the inequality (23) implies the global exponential stability of the system (21). Since the reference input $y_{c}(t)$ is bounded, from the boundedness of $\boldsymbol{e}(t)$ and $\boldsymbol{x}_{m}(t)$ the boundedness of $\boldsymbol{x}(t)$ follows. Therefore, $\alpha(t, \boldsymbol{x})$ is bounded, implying the boundedness of $\boldsymbol{z}^{c}(t)$. Therefore, the state $\boldsymbol{x}_{a}^{c}(t)$ is bounded. Then, the boundedness of internal state $\boldsymbol{\xi}_{a}^{c}(t)$ follows from the input to state stability of the actuator's internal dynamics.

Remark 1 We notice that the control signal $u_{b}(t)$ is comprised of two parts. The first part does not contain high gain and is designed from the perspective of retaining the slow mode of the actuator's dynamics, since it cannot be altered without a high magnitude input signal. The second part is inversely proportional to the small parameter $\varepsilon$ and is designed to cancel interconnecting terms. This part is a potential source of the high magnitude command that can exceed the actuator's physical limits.

Next, we apply singular perturbation method to the cascaded error system (19), and introduce a new (slow) time variable $\tau=\varepsilon^{r} t$. Ignoring the actuator's input to state stable internal dynamics and substituting $\tau$ results in the following system

$$
\begin{aligned}
\varepsilon^{r} \frac{\boldsymbol{x}(\tau)}{d \tau} & =\boldsymbol{f}(\tau, \boldsymbol{x})+\boldsymbol{g} \eta_{r}(\tau) \\
\frac{d \eta_{r}(\tau)}{d \tau} & =-\boldsymbol{k}_{a}^{\top} \boldsymbol{x}_{a}(\tau)+v(\tau)-\frac{d \alpha_{r}\left(\tau, \boldsymbol{x}_{p}\right)}{d \tau} .
\end{aligned}
$$

This is a singular perturbation problem. It is in the standard form if the algebraic equation

$$
\boldsymbol{f}(\tau, \boldsymbol{x})+\boldsymbol{g} \eta_{r}(\tau)=0
$$

has isolated roots. Here we are concerned about the conditions of the existence of the isolated roots in general. For our purposes it is sufficient to note that the equation (25) has a root $\boldsymbol{x}^{*}=\left[\boldsymbol{e}=0, \eta_{1}=\right.$ 
$0, \ldots, \eta_{r-2}=0, \eta_{r-1}=\lambda_{r-1}^{-1} \eta_{r}(t)$, which we denote by $\boldsymbol{x}^{*}=\boldsymbol{h}\left(t, \eta_{r}\right)$. Introducing a change of variables $\boldsymbol{\zeta}(\tau)=\boldsymbol{x}(\tau)-\boldsymbol{h}\left(t, \eta_{r}\right)$, the boundary layer system is obtained from the first equation in (24) as follows

$$
\frac{d \boldsymbol{\zeta}(t)}{d t}=\boldsymbol{f}(t, \boldsymbol{\zeta}(t)) .
$$

It is easy to see that the system (26) has an equilibrium at the origin. The reduced system takes the form

$$
\frac{d \eta_{r}(\tau)}{d \tau}=-\boldsymbol{k}_{a}^{\top} \boldsymbol{x}_{a}(\tau)+v(\tau)-\left.\frac{d \alpha_{r}\left(\tau, \boldsymbol{x}_{p}\right)}{d \tau}\right|_{\boldsymbol{e}=\boldsymbol{h}\left(\tau, \eta_{r}\right)} .
$$

Unlike the previous case, here the control signal $v(\tau)$ is designed to stabilize only the reduced system $(27)$ and has the form

$$
v_{p}(t)=\boldsymbol{k}_{a}^{\top} \boldsymbol{x}_{a}(t)-\lambda_{r} \eta_{r}(t)+\left.\varepsilon^{-r} \dot{\alpha}_{r}\left(t, \boldsymbol{x}_{p}\right)\right|_{\boldsymbol{e}=\boldsymbol{h}\left(t, \eta_{r}\right)} .
$$

Applying the control signal $v_{p}(t)$ results in the following closed-loop reduced systems in the real time scale $t$

$$
\dot{\eta}_{r}(t)=-\varepsilon^{r} \lambda_{r} \eta_{r}(t),
$$

which has a globally exponentially stable equilibrium at the origin $\left(\eta_{r}=0\right)$.

Lemma 2 Under the control action (28), the singular perturbation problem (24) with initial conditions $\boldsymbol{e}_{0}, \eta_{1}(0), \ldots, \eta_{r}(0)$ has a unique solution $e(t, \varepsilon), \eta_{1}(t, \varepsilon), \ldots, \eta_{r}(t, \varepsilon)$, which satisfies the relationship

$$
\eta_{r}(t, \varepsilon)-\bar{\eta}_{r}(t)=O(\varepsilon)
$$

for all $t \geq 0$, where $\bar{\eta}_{r}(t)$ is the unique solution of the reduced system (27) with the initial condition $\eta_{r}(o)=$ $z_{r}^{c}(0)-\alpha_{r}\left(0, \boldsymbol{x}_{0}\right)$. Further, there exists a time instance $T^{*}$ such that the relationship

$$
\boldsymbol{x}(t, \varepsilon)=O(\varepsilon) .
$$

holds for all $t \geq T^{*}$. Moreover, all closed-loop signals are bounded.

Proof. Consider the candidate Lyapunov function

$$
V_{3}(\boldsymbol{e}(t), \eta(t))=V_{1}(\boldsymbol{e}(t))+\eta_{1}^{2}(t)+\cdots+\eta_{r-1}^{2}(t) .
$$

Its derivative along the trajectories of boundary layer system (26) can be easily computed to satisfy the inequality

$$
\dot{V}_{3}(\boldsymbol{\zeta}(t), z(t))<-\mu V_{3}(\boldsymbol{\zeta}(t), z(t)),
$$

with $\mu=\lambda_{\max }^{-1}(P) \min \left[\lambda_{\min }^{-1}(Q), \lambda_{1}, \ldots, \lambda_{r-1}\right]$, which implies the global exponential stability of the boundary layer system. The smoothness and boundedness conditions of Theorem 1 follow from the assumptions imposed in this section. Therefore, from Theorem 1 it follows that (30) holds for all $t \geq 0$, and there exists a time instance $T(\varepsilon)$ such that the relationship

$$
\boldsymbol{x}(t, \varepsilon)-\boldsymbol{h}\left(t, \eta_{r}(t)\right)=O(\varepsilon)
$$

holds for all $t \geq T(\varepsilon)$. From the exponential stability of (29) it follows that there exists a time instant $T_{1}$ such that $\eta_{r}(t)=O(\varepsilon)$ for $t \geq T_{1}$. Then, from the definition of $\boldsymbol{h}(t, \boldsymbol{x}(t))$ it follows that the relationship $\boldsymbol{h}(t, \boldsymbol{x}(t))=O(\varepsilon)$ holds for $t \geq T_{1}$. Therefore (33) holds for $t \geq T^{*}$, where $T^{*}=\max \left[T(\epsilon), T_{1}\right]$. From the above relationship and the boundedness of the reference model (10) it follows that the plant's state $\boldsymbol{x}_{p}(t)$ is bounded, implying the boundedness of the stabilizing functions $\alpha_{i}\left(t, \boldsymbol{x}_{p}\right), i=1, \ldots, r$. Since $\boldsymbol{\eta}(t)$ is bounded, the boundedness of $\boldsymbol{z}^{c}(t)$ follows, implying also the boundedness of the actuator's state $\boldsymbol{x}_{a}^{c}(t)$. From the properties of the actuator's internal dynamics it follows that the internal state $\boldsymbol{\xi}_{a}^{c}(t)$ is bounded as well.

Remark 2 Instead of the exponential tracking achieved by the backstepping control $v_{b}(t)$ (20), the control law $v_{p}(t)$ (28) achieves only $\varepsilon$-tracking for the original error dynamics, with a transient time greater than $\left.T^{*}\right]$. However, the latter control signal has a smaller magnitude than the former one. To see this, we first notice from the continuity of the function $\dot{\alpha}(t, \boldsymbol{x})$ that $\dot{\alpha}(t, x)-\left.\dot{\alpha}(t, x)\right|_{\boldsymbol{e}=\overline{\boldsymbol{h}}(t, z)}=O(\varepsilon)$. Taking into account the relationship (80), we can write

$$
v_{b}(t)-v_{p}(t)=O(\varepsilon)-\varepsilon^{-r} \eta_{r-1}(t)
$$

which implies that the two control signals differ by the high gain term $\varepsilon^{-r} \eta_{r-1}(t)$, which is inversely proportional to $\varepsilon^{r}$ and comes from $v_{b}(t)(20)$. 


\section{Control design for uncertain systems}

In this section, we solve the same control problem assuming uncertain plant's and actuator's dynamics that are also subject to external disturbances $d_{p}(t)$ and $d_{a}(t)$ respectively, which have bounded derivatives. To be precise, we assume that the parameter $\omega$, matrices $A$ and $A_{a}$, vector $\boldsymbol{b}_{a}$, and continuously differentiable functions $f_{p}\left(\boldsymbol{x}_{p}\right), g_{p}\left(\boldsymbol{x}_{p}\right), g_{a}\left(y_{a}\right)$ are unknown. The control objective is to design a control law that uses the plant's state $\boldsymbol{x}_{p}(t)$ and the actuator's output $y_{a}(t)$ for feedback to achieve tracking of the reference model (10).

As in conventional adaptive control, using the universal approximation theorem, we approximate the unknown functions by radial basis functions networks on compact sets $\Omega_{x}$ and $\Omega_{y_{a}}$

$$
\begin{aligned}
f_{p}\left(\boldsymbol{x}_{p}\right) & =\boldsymbol{w}_{f}^{\top} \boldsymbol{\varphi}_{f}\left(\boldsymbol{x}_{p}\right)+\delta_{f}\left(\boldsymbol{x}_{p}\right) \\
g_{p}\left(\boldsymbol{x}_{p}\right) & =\boldsymbol{w}_{g}^{\top} \boldsymbol{\varphi}_{g}\left(\boldsymbol{x}_{p}\right)+\delta_{g}\left(\boldsymbol{x}_{P}\right) \\
g_{a}\left(y_{a}, \boldsymbol{p}_{a}\right) & =\boldsymbol{w}_{a}^{\top} \boldsymbol{\varphi}_{a}\left(y_{a}\right)+\delta_{a}\left(y_{a}\right),
\end{aligned}
$$

where $\delta_{f}\left(\boldsymbol{x}_{p}\right), \delta_{g}\left(\boldsymbol{x}_{p}\right)$ and $\delta_{a}\left(y_{a}\right)$ are the approximation errors, uniformly bounded by positive constants $\delta_{f}^{*}$, $\delta_{g}^{*}$ and $\delta_{a}^{*}$ respectively. Using the relationships (36), the error dynamics (13) can be written as

$$
\dot{\boldsymbol{e}}(t)=A_{m} \boldsymbol{e}(t)+\boldsymbol{b}_{p}\left[\boldsymbol{k}^{\top} \boldsymbol{x}_{p}(t)+\boldsymbol{w}_{f}^{\top} \boldsymbol{\varphi}_{f}\left(\boldsymbol{x}_{p}\right)+\boldsymbol{w}_{g}^{\top} \boldsymbol{\varphi}_{g}\left(\boldsymbol{x}_{p}\right) y_{a}(t)+\sigma_{p}\left(t, \boldsymbol{x}_{p}, y_{a}\right)-\nu y_{c}(t)\right],
$$

where $\sigma_{p}\left(t, \boldsymbol{x}_{p}, y_{a}\right)=\delta_{f}\left(\boldsymbol{x}_{p}(t)\right)+\delta_{g}\left(\boldsymbol{x}_{p}(t)\right) y_{a}(t)+d_{p}(t)$ is the combined disturbance term, which is uniformly bounded by a positive constant $\sigma_{1}^{*}$ as long as $\boldsymbol{x}_{p}(t) \in \Omega_{x}$ and $y_{a}(t) \in \Omega_{y}$.

In this section, we assume that the actuator's dynamics has a relative degree $m-r+1$ and is represented in the following observer canonical form

$$
\begin{aligned}
\varepsilon^{-1} \dot{\boldsymbol{x}}_{a}(t) & =A_{0} \boldsymbol{x}_{a}(t)-\boldsymbol{q} y_{a}(t)+\boldsymbol{b}_{a}\left[u(t)+g_{a}\left(y_{a}(t), \boldsymbol{p}_{a}\right)+d_{a}(t)\right] \\
y_{a}(t) & =\boldsymbol{c}_{0}^{\top} \boldsymbol{x}_{a}(t)
\end{aligned}
$$

where $A_{0}$ and $\boldsymbol{c}_{0}$ are defined similar to definitions in the previous section with $r$ replaced by $m, \boldsymbol{q} \in \mathbb{R}^{m}$ is an

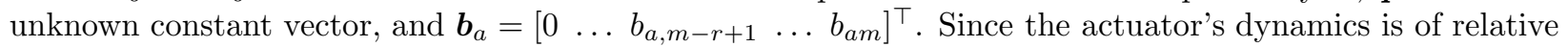
degree $m-r+1$, the $b_{a, m-r+1}$ is not zero. Let it be positive. Taking into account the approximation in (36), the dynamics of $\boldsymbol{x}_{a}(t)$ is written as

$$
\varepsilon^{-1} \dot{\boldsymbol{x}}_{a}(t)=A_{0} \boldsymbol{x}_{a}(t)-\boldsymbol{q} y_{a}(t)+\boldsymbol{b}_{a}\left[u(t)+\boldsymbol{w}_{a}^{\top} \boldsymbol{\varphi}_{a}\left(y_{a}\right)+\sigma_{a}\left(t, y_{a}\right)\right] .
$$

where $\sigma_{a}\left(t, y_{a}\right)=\delta_{a}\left(y_{a}\right)+d_{a}(t)$ is the combined disturbance term, which is uniformly bounded by a positive constant $\sigma_{a}^{*}$ as long as $y_{a} \in \Omega_{y_{a}}$.

To avoid generating high gain control signals we again apply the singular perturbation method. However, the method cannot be directly applied, since the exponential stability properties for the resulting boundary layer and reduced systems cannot be establish in the adaptive control framework without parameter convergence. The latter can be guaranteed only for sufficiently rich regressor or input signals, which in general is difficult to verify a priory. As an intermediate step, we follow the approach from Ref. ${ }^{4}$ and design suitable prediction models for which the exponential stability properties can be guaranteed, and hence Tikhonov's theorem can be applied. The control objective is met when the closeness of the prediction models to the corresponding dynamics is established. This will be done by the choice of adaptive laws independent of the control design.

\section{A. Prediction models}

We consider the following prediction model for the plant's tracking error dynamics

$$
\dot{\hat{\boldsymbol{e}}}(t)=A_{m} \hat{\boldsymbol{e}}(t)+\boldsymbol{b}\left[\hat{\boldsymbol{k}}^{\top}(t) \boldsymbol{x}(t)+\hat{\boldsymbol{w}}_{f}^{\top}(t) \boldsymbol{\varphi}_{f}(\boldsymbol{x})+\hat{\boldsymbol{w}}_{g}^{\top}(t) \boldsymbol{\varphi}_{g}(\boldsymbol{x}) \hat{y}_{a}(t)+\hat{\sigma}_{p}(t) \operatorname{sign}(\psi(t))-\nu y_{c}(t)\right],
$$

where the initial conditions $\hat{\boldsymbol{e}}(0)$ is chosen identical with $\boldsymbol{e}(0), \psi(t)=[\boldsymbol{e}(t)-\hat{\boldsymbol{e}}(t)]^{\top} \boldsymbol{P} \boldsymbol{b}_{p}$, and all variables with "hat" are the estimates of the corresponding variables without "hat", and are available for the control 
design. Let the prediction errors be $\tilde{\boldsymbol{e}}(t)=\boldsymbol{e}(t)-\hat{\boldsymbol{e}}(t)$. Its dynamics can be written in the form

$$
\begin{aligned}
\dot{\tilde{\boldsymbol{e}}}(t) & =A_{m} \tilde{\boldsymbol{e}}(t)+\boldsymbol{b}_{p}\left[\tilde{\boldsymbol{k}}^{\top}(t) \boldsymbol{x}_{p}(t)+\tilde{\boldsymbol{w}}_{f}^{\top}(t) \boldsymbol{\varphi}_{f}\left(\boldsymbol{x}_{p}\right)+\tilde{\boldsymbol{w}}_{g}^{\top}(t) \boldsymbol{\varphi}_{g}\left(\boldsymbol{x}_{p}\right) y_{a}(t)\right. \\
& \left.+\hat{\boldsymbol{w}}_{g}^{\top}(t) \boldsymbol{\varphi}_{g}\left(\boldsymbol{x}_{p}\right) \tilde{y}_{a}(t)+\sigma_{p}\left(t, \boldsymbol{x}_{p}, y_{a}\right)-\hat{\sigma}_{p}(t) \operatorname{sign}(\psi(t))\right],
\end{aligned}
$$

where we introduce parameter estimation errors $\tilde{\boldsymbol{k}}(t)=\boldsymbol{k}-\hat{\boldsymbol{k}}(t), \tilde{\boldsymbol{w}}_{f}(t)=\boldsymbol{w}_{f}-\hat{\boldsymbol{w}}_{f}(t), \tilde{\boldsymbol{w}}_{g}(t)=\boldsymbol{w}_{g}-\hat{\boldsymbol{w}}_{g}(t)$, $\tilde{\sigma}_{p}(t)=\sigma_{p}^{*}-\hat{\sigma}_{p}(t)$.

To be able to introduce a suitable prediction model for the actuator dynamics in (39), we re-parameterize them following conventional adaptive observer-estimator scheme (see for example ${ }^{9,15}$ ) and introduce filters

$$
\begin{aligned}
\varepsilon^{-1} \dot{\boldsymbol{\beta}}_{1}(t) & =\left(A_{0}-\boldsymbol{b}^{0} \boldsymbol{k}_{0}^{\top}\right) \boldsymbol{\beta}_{1}(t)+\boldsymbol{b}^{0} y_{a}(t), \quad \boldsymbol{\beta}_{1}(0)=0 \\
\varepsilon^{-1} \dot{\boldsymbol{\beta}}_{2}(t) & =\left(A_{0}-\boldsymbol{b}^{0} \boldsymbol{k}_{0}^{\top}\right) \boldsymbol{\beta}_{2}(t)+\boldsymbol{b}^{0} u(t), \quad \boldsymbol{\beta}_{2}(0)=0 \\
\varepsilon^{-1} \dot{\boldsymbol{\beta}}_{3}(t) & =\left(A_{0}-\boldsymbol{b}^{0} \boldsymbol{k}_{0}^{\top}\right) \boldsymbol{\beta}_{3}(t)+\boldsymbol{b}^{0} \sigma_{a}\left(t, y_{a}\right), \quad \boldsymbol{\beta}_{3}(0)=0 \\
\varepsilon^{-1} \dot{\Xi}(t) & =\left(A_{0}-\boldsymbol{b}^{0} \boldsymbol{k}_{0}^{\top}\right) \Xi(t)+\boldsymbol{b}^{0} \boldsymbol{\varphi}_{a}^{\top}\left(y_{a}\right), \quad \Xi(0)=0,
\end{aligned}
$$

where $\boldsymbol{b}^{0}=\left[\begin{array}{lllllll}0 & \ldots & 1 & b_{r+1}^{0} & \ldots & b_{m}^{0}\end{array}\right]^{\top}$ is chosen such that the pair $\left(A_{0}, \boldsymbol{b}^{0}\right)$ is controllable and the polynomial $s^{m-r}+b_{r+1}^{0} s^{m-r-1}+\cdots+b_{m}^{0}$ is Hurwitz, and the vector $\boldsymbol{k}_{0}$ is chosen such that the matrix $A_{0}-\boldsymbol{b}^{0} \boldsymbol{k}_{0}^{\top}$ is Hurwitz. The actuator's output $y_{a}(t)$ is represented as

$$
y_{a}(t)=\boldsymbol{\vartheta}_{1}^{\top} \boldsymbol{\omega}_{1}(t)+\boldsymbol{\vartheta}_{2}^{\top} \boldsymbol{\omega}_{2}(t)+\boldsymbol{w}_{a}^{\top} \boldsymbol{\omega}_{3}(t)+\sigma_{f}(t),
$$

where $\boldsymbol{\vartheta}_{1} \in \mathbb{R}^{m}$ and $\boldsymbol{\vartheta}_{1}=\left[\begin{array}{lll}b_{a m} & \ldots & b_{a, m-r+1}\end{array}\right]^{\top} \in \mathbb{R}^{r}$ are unknown constant parameters to be estimated online, $\boldsymbol{\omega}_{1}(t)=\boldsymbol{\beta}_{1}(t), \boldsymbol{\omega}_{2}(t)=\left[\begin{array}{lll}\beta_{21}(t) & \ldots & \beta_{2 r}(t)\end{array}\right]^{\top}, \boldsymbol{\omega}_{3}(t)=\boldsymbol{c}_{0}^{\top} \Xi(t)$ and $\sigma_{f}(t)=\boldsymbol{c}_{0}^{\top} \boldsymbol{\beta}_{3}(t)$. Since the filters are stable from the boundedness of the inputs the boundedness of the outputs follow. That is $\sigma_{f}(t)$ is bounded by some positive constant $\sigma_{f}^{*}$ since $\sigma_{a}\left(t, y_{a}\right)$ is bounded and the vector function $\boldsymbol{\omega}_{3}(t)$ is bounded since the RBFs are bounded. The prediction model for the actuator dynamics in (39) is introduced as

$$
\hat{y}_{a}(t)=\hat{\boldsymbol{\vartheta}}_{1}^{\top}(t) \boldsymbol{\omega}_{1}(t)+\hat{\boldsymbol{\vartheta}}_{2}^{\top}(t) \boldsymbol{\omega}_{2}(t)+\hat{\boldsymbol{w}}_{a}^{\top}(t) \boldsymbol{\omega}_{3}(t)+\hat{\sigma}_{f}(t) \operatorname{sign}(\psi(t)),
$$

where again the variables with "hat" are the estimates of the corresponding variables without "hat", and $\tilde{y}_{a}(t)=y_{a}(t)-\hat{y}_{a}(t)$ is the prediction error, which is given by the equation

$$
\tilde{y}_{a}(t)=\tilde{\boldsymbol{\vartheta}}_{1}^{\top}(t) \boldsymbol{\omega}_{1}(t)+\tilde{\boldsymbol{\vartheta}}_{2}^{\top}(t) \boldsymbol{\omega}_{2}(t)+\tilde{\boldsymbol{w}}_{a}^{\top}(t) \boldsymbol{\omega}_{3}(t)+\sigma_{f}(t)-\hat{\sigma}_{f}(t) \operatorname{sign}(\psi(t)),
$$

where $\tilde{\boldsymbol{\vartheta}}_{1}(t)=\boldsymbol{\vartheta}_{1}-\hat{\boldsymbol{\vartheta}}_{1}(t), \tilde{\boldsymbol{\vartheta}}_{2}(t)=\boldsymbol{\vartheta}_{2}-\hat{\boldsymbol{\vartheta}}_{2}(t)$ and $\tilde{\boldsymbol{w}}_{a}(t)=\boldsymbol{w}_{a}-\hat{\boldsymbol{w}}_{a}(t)$.

Since $\boldsymbol{g}\left(x_{p}\right)$ is assumed to be positive, the neural network approximation (36) can be chosen such that the unknown weights $\boldsymbol{w}_{g}$ are positive. ${ }^{11}$ The RBFs can be chosen to be positive as well. Then the product $\boldsymbol{w}_{g}^{\top}(t) \boldsymbol{\varphi}_{g}\left(\boldsymbol{x}_{p}\right)$ is always positive. Therefore, it is reasonable to design the adaptive law such that all components of $\hat{\boldsymbol{w}}_{g}(t)$ are positive. This will be done by means of the projection operator. ${ }^{12}$ In the same fashion, since $\vartheta_{2, r}>0$, the adaptive law for $\hat{\vartheta}_{2, r}(t)$ is designed to keep it positive. Having this in mind, we design the adaptive laws for the estimates $\hat{\boldsymbol{k}}(t), \hat{\boldsymbol{w}}_{f}(t), \hat{\boldsymbol{w}}_{g}(t), \hat{\sigma}_{p}(t)$ as follows

$$
\begin{aligned}
\dot{\hat{\sigma}}_{p}(t) & =\gamma|\psi(t)| \\
\dot{\hat{\boldsymbol{k}}}(t) & =\gamma \boldsymbol{x}_{p}(t) \psi(t) \\
\dot{\hat{\boldsymbol{w}}}_{f}(t) & =\gamma \boldsymbol{\varphi}_{f}(\boldsymbol{x}) \psi(t) \\
\dot{\hat{\boldsymbol{w}}}_{g}(t) & =\gamma \Pi\left\{\hat{\boldsymbol{w}}_{g}(t), y_{a}(t) \boldsymbol{\varphi}_{g}\left(\boldsymbol{x}_{p}\right) \psi(t)\right\},
\end{aligned}
$$

where $\gamma>0$ is the adaptation rate, $\Pi\{\cdot, \cdot\}$ denotes the projection operator, ${ }^{12}$ introduced here to keep the estimates $\hat{\boldsymbol{w}}_{g}(t)$ bounded away from zero.

The adaptive law for $\hat{\vartheta}_{2, r}(t)$ is defined by means of the projection operator.

$$
\dot{\hat{\vartheta}}_{2, r}(t)=\gamma \Pi\left(\hat{\vartheta}_{2 r}(t), \tilde{y}_{a}(t) \omega_{2 r}(t)+\hat{\boldsymbol{w}}_{g}^{\top}(t) \boldsymbol{\varphi}_{g}(\boldsymbol{x}) \psi(t) \omega_{2 r}(t)\right) .
$$


The remaining adaptive laws are

$$
\begin{aligned}
\dot{\hat{\boldsymbol{\vartheta}}}_{1}(t) & =\gamma \tilde{y}_{a}(t) \boldsymbol{\omega}_{1}(t)+\gamma \hat{\boldsymbol{w}}_{g}^{\top}(t) \boldsymbol{\varphi}_{g}(\boldsymbol{x}) \psi(t) \boldsymbol{\omega}_{1}(t) \\
\dot{\hat{\vartheta}}_{2 i}(t) & =\gamma\left(\tilde{y}_{a}(t) \omega_{2 i}(t)+\hat{\boldsymbol{w}}_{g}^{\top}(t) \boldsymbol{\varphi}_{g}(\boldsymbol{x}) \psi(t) \omega_{2 i}(t)\right), \quad i=1, \ldots, r-1 \\
\dot{\hat{\boldsymbol{w}}}_{a}(t) & =\gamma \tilde{y}_{a}(t) \boldsymbol{\omega}_{3}(t)+\gamma \hat{\boldsymbol{w}}_{g}^{\top}(t) \boldsymbol{\varphi}_{g}(\boldsymbol{x}) \psi(t) \boldsymbol{\omega}_{3}(t) \\
\dot{\hat{\sigma}}_{f}(t) & =\gamma\left|\tilde{y}_{a}(t)\right|+\gamma \hat{\boldsymbol{w}}_{g}^{\top}(t) \boldsymbol{\varphi}_{g}(\boldsymbol{x})|\psi(t)|
\end{aligned}
$$

where the projection operator $\Pi(\cdot, \cdot)$ is introduced to keep $\hat{\omega}(t)$ positive.

The following lemma guarantees the closeness of the prediction models to the corresponding dynamics.

Lemma 3 The adaptive laws (46), (47) and (48) guarantee boundedness of the estimates $\hat{\boldsymbol{k}}_{(}(t), \hat{\boldsymbol{w}}_{f}(t), \hat{\boldsymbol{w}}_{g}(t), \hat{\sigma}_{p}(t)$ as well as $\hat{\boldsymbol{\vartheta}}_{1}(t), \hat{\boldsymbol{w}}_{a}(t), \hat{\boldsymbol{\vartheta}}_{1}(t), \hat{\sigma}_{f}(t)$ and the prediction errors $\tilde{\boldsymbol{e}}(t)$ and $\tilde{y}_{a}(t)$. Moreover, $\tilde{\boldsymbol{e}}(t) \in \mathcal{L}_{2}$ and $\tilde{y}_{a}(t) \in \mathcal{L}_{2}$.

Proof. Consider the following candidate Lyapunov function

$$
\begin{aligned}
V_{4}(t) & =\tilde{\boldsymbol{e}}^{\top}(t) P \tilde{\boldsymbol{e}}(t)+\gamma^{-1}\left[\left(\sigma_{p}^{*}-\hat{\sigma}_{p}(t)\right)^{2}+\tilde{\boldsymbol{k}}^{\top}(t) \tilde{\boldsymbol{k}}(t)+\tilde{\boldsymbol{w}}_{f}^{\top}(t) \tilde{\boldsymbol{w}}_{f}(t)+\tilde{\boldsymbol{w}}_{g}^{\top}(t) \tilde{\boldsymbol{w}}_{g}(t)\right. \\
& \left.+\tilde{\boldsymbol{\vartheta}}^{\top}(t) \tilde{\boldsymbol{\vartheta}}_{1}(t)+\tilde{\boldsymbol{\vartheta}}_{2}^{\top}(t) \tilde{\boldsymbol{\vartheta}}_{2}(t)+\tilde{\boldsymbol{w}}_{a}^{\top}(t) \tilde{\boldsymbol{w}}_{a}(t)+\left(\sigma_{f}^{*}-\hat{\sigma}_{f}(t)\right)^{2}\right] .
\end{aligned}
$$

The derivative of $V_{4}(t)$ is computed along the trajectories of systems (41), and (46) and (48).

$$
\begin{aligned}
& \dot{V}_{4}(t)=\tilde{\boldsymbol{e}}^{\top}(t)\left[A_{m}^{\top} P+P A_{m}\right] \tilde{\boldsymbol{e}}(t)+2 \tilde{\boldsymbol{e}}^{\top}(t) P \boldsymbol{b}_{p}\left[\tilde{\boldsymbol{k}}^{\top}(t) \boldsymbol{x}_{p}(t)+\tilde{\boldsymbol{w}}_{f}^{\top}(t) \boldsymbol{\varphi}_{f}\left(\boldsymbol{x}_{p}\right)+\tilde{\boldsymbol{w}}_{g}^{\top}(t) \boldsymbol{\varphi}_{g}\left(\boldsymbol{x}_{p}\right) y_{a}(t)\right. \\
& +\hat{\boldsymbol{w}}_{g}^{\top}(t) \boldsymbol{\varphi}_{g}\left(\boldsymbol{x}_{p}\right)\left[\tilde{\boldsymbol{\vartheta}}_{1}^{\top}(t) \boldsymbol{\omega}_{1}(t)+\tilde{\boldsymbol{\vartheta}}_{2}^{\top}(t) \boldsymbol{\omega}_{2}(t)+\tilde{\boldsymbol{w}}_{a}^{\top}(t) \boldsymbol{\omega}_{3}(t)+\sigma_{f}(t)-\hat{\sigma}_{f}(t) \operatorname{sign}\left(\tilde{y}_{a}(t)\right)\right]+\sigma_{p}\left(t, \boldsymbol{x}_{p}, y_{a}\right) \\
& \left.-\hat{\sigma}_{p}(t) \operatorname{sign}(\psi(t))\right]+2 \gamma^{-1}\left[-\left(\sigma_{p}^{*}-\hat{\sigma}_{p}(t)\right) \dot{\hat{\sigma}}_{p}(t)-\tilde{\boldsymbol{k}}^{\top}(t) \dot{\hat{\boldsymbol{k}}}(t)-\tilde{\boldsymbol{w}}_{f}^{\top}(t) \dot{\hat{\boldsymbol{w}}}_{f}(t)-\tilde{\boldsymbol{w}}_{g}^{\top}(t) \dot{\hat{\boldsymbol{w}}}_{g}(t)\right. \\
& \left.-\tilde{\boldsymbol{\vartheta}}_{1}^{\top}(t) \dot{\hat{\boldsymbol{\vartheta}}}_{1}(t)-\tilde{\boldsymbol{\vartheta}}_{2}(t) \dot{\hat{\boldsymbol{\vartheta}}}_{2}(t)-\tilde{\boldsymbol{w}}_{a}^{\top}(t) \dot{\tilde{\boldsymbol{w}}}_{a}(t)-\left(\sigma_{f}^{*}-\hat{\sigma}_{f}(t)\right) \dot{\sigma}_{f}(t)\right] \\
& =-\tilde{\boldsymbol{e}}^{\top}(t) Q \tilde{\boldsymbol{e}}(t)+2 \tilde{\boldsymbol{w}}_{g}^{\top}(t)\left[\psi(t) \boldsymbol{\varphi}_{g}\left(\boldsymbol{x}_{p}\right) y_{a}(t)-\gamma^{-1} \dot{\hat{\boldsymbol{w}}}_{g}(t)\right]+2 \tilde{\boldsymbol{w}}_{f}^{\top}(t)\left[\psi(t) \boldsymbol{\varphi}_{f}\left(\boldsymbol{x}_{p}\right)-\gamma^{-1} \dot{\hat{\boldsymbol{w}}}_{f}(t)\right] \\
& +2 \tilde{\boldsymbol{k}}^{\top}(t)\left[\psi(t) \boldsymbol{x}_{p}(t)-\gamma^{-1} \dot{\hat{\boldsymbol{k}}}(t)\right]+2\left[\psi(t) \sigma_{p}\left(t, \boldsymbol{x}_{p}, y_{a}\right)-\hat{\sigma}_{p}(t)|\psi(t)|-\gamma^{-1}\left(\sigma_{p}^{*}-\hat{\sigma}_{p}(t)\right) \dot{\hat{\sigma}}_{p}(t)\right] \\
& +2 \tilde{\boldsymbol{\vartheta}}_{1}^{\top}(t)\left[\psi(t) \hat{\boldsymbol{w}}_{g}^{\top}(t) \boldsymbol{\varphi}_{g}\left(\boldsymbol{x}_{p}\right) \boldsymbol{\chi}_{1}(t)-\gamma^{-1} \dot{\hat{\boldsymbol{\vartheta}}}_{1}(t)\right]+2 \tilde{\boldsymbol{w}}_{a}^{\top}(t)\left[\psi(t) \hat{\boldsymbol{w}}_{g}^{\top}(t) \boldsymbol{\varphi}_{g}\left(\boldsymbol{x}_{p}\right) \boldsymbol{\omega}_{3}(t)-\gamma^{-1} \dot{\hat{\boldsymbol{w}}}_{a}(t)\right] \\
& +2 \tilde{\boldsymbol{\vartheta}}_{2}(t)\left[\psi(t) \hat{\boldsymbol{w}}_{g}^{\top}(t) \boldsymbol{\varphi}_{g}\left(\boldsymbol{x}_{p}\right) \boldsymbol{\omega}_{2}(t)-\gamma^{-1} \dot{\hat{\boldsymbol{\vartheta}}}_{2}(t)\right]+2 \psi(t) \hat{\boldsymbol{w}}_{g}^{\top}(t) \boldsymbol{\varphi}_{g}\left(\boldsymbol{x}_{p}\right) \sigma_{f}(t) \\
& \left.-2 \hat{\boldsymbol{w}}_{g}^{\top}(t) \boldsymbol{\varphi}_{g}\left(\boldsymbol{x}_{p}\right) \hat{\sigma}_{f}^{*}|\psi(t)|+2 \hat{\boldsymbol{w}}_{g}^{\top}(t) \boldsymbol{\varphi}_{g}\left(\boldsymbol{x}_{p}\right)\left[\sigma_{f}^{*}-\hat{\sigma}_{f}(t)\right]|\psi(t)|-2 \gamma^{-1}\left(\sigma_{f}^{*}-\hat{\sigma}_{f}(t)\right) \dot{\hat{\sigma}}_{f}(t)\right] \text {. }
\end{aligned}
$$

Substituting the adaptive laws and taking into account the properties of the projection operator ${ }^{12}$

$$
\begin{gathered}
\tilde{\boldsymbol{w}}_{f}^{\top}(t)\left[y_{a}(t) \boldsymbol{\varphi}_{g}\left(\boldsymbol{x}_{p}\right) \psi(t)-\Pi\left\{\hat{\boldsymbol{w}}_{g}(t), y_{a}(t) \boldsymbol{\varphi}_{g}(\boldsymbol{x}) \psi(t)\right\}\right] \leq 0 \\
\tilde{\vartheta}_{2 r}(t)\left[\tilde{y}_{a}(t) \omega_{2 r}(t)+\hat{\boldsymbol{w}}_{g}(t) \boldsymbol{\varphi}_{g}(\boldsymbol{x}) \psi(t) \omega_{2 r}(t)-\Pi\left\{\hat{\vartheta}_{2 r}(t), \tilde{y}_{a}(t) \omega_{2 r}(t)+\hat{\boldsymbol{w}}_{g}^{\top} \boldsymbol{\varphi}_{g}(\boldsymbol{x}) \psi(t) \omega_{2 r}(t)\right\}\right] \leq 0,
\end{gathered}
$$

we obtain

$$
\begin{aligned}
\dot{V}_{4}(t) & \leq-\tilde{\boldsymbol{e}}^{\top}(t) Q \tilde{\boldsymbol{e}}(t)+2 \psi(t) \sigma_{p}\left(t, \boldsymbol{x}_{p}, y_{a}\right)-2 \hat{\sigma}_{p}(t)|\psi(t)|-2 \tilde{y}_{a}(t)\left[\tilde{\boldsymbol{\vartheta}}_{1}^{\top}(t) \boldsymbol{\omega}_{1}(t)+\tilde{\boldsymbol{\vartheta}}_{2}^{\top}(t) \boldsymbol{\omega}_{2}(t)+\tilde{\boldsymbol{w}}_{a}^{\top}(t) \boldsymbol{\omega}_{3}(t)\right] \\
& +2 \psi(t) \hat{\boldsymbol{w}}_{g}^{\top}(t) \boldsymbol{\varphi}_{g}\left(\boldsymbol{x}_{p}\right) \sigma_{f}(t)-2 \hat{\boldsymbol{w}}_{g}^{\top}(t) \boldsymbol{\varphi}_{g}\left(\boldsymbol{x}_{p}\right) \sigma_{f}^{*}|\psi(t)|-\left(\sigma_{f}^{*}-\hat{\sigma}_{f}(t)\right)\left|\tilde{y}_{a}(t)\right| .
\end{aligned}
$$

We notice that $\psi(t) \sigma_{p}\left(t, \boldsymbol{x}_{p}, y_{a}\right)-\sigma_{p}^{*}|\psi(t)| \leq 0$, and $\psi(t) \hat{\boldsymbol{w}}_{g}^{\top}(t) \boldsymbol{\varphi}_{g}\left(\boldsymbol{x}_{p}\right) \sigma_{f}(t)-\hat{\boldsymbol{w}}_{g}^{\top}(t) \boldsymbol{\varphi}_{g}\left(\boldsymbol{x}_{p}\right) \sigma_{f}^{*}|\psi(t)| \leq 0$, since $\hat{\boldsymbol{w}}_{g}^{\top}(t) \boldsymbol{\varphi}_{g}\left(\boldsymbol{x}_{p}\right)$ is positive by construction. Moreover, from the adaptive law in (48) it follows that $\hat{\sigma}_{f}(t) \geq 0$ as long as $\hat{\sigma}_{f}(0) \geq 0$. Therefore the following inequality holds

$$
\left(\sigma_{f}^{*}-\hat{\sigma}_{f}(t)\left|\tilde{y}_{a}(t)\right| \geq \sigma_{f}(t) \tilde{y}_{a}(t)-\hat{\sigma}_{f}(t) \operatorname{sign}(\psi(t)) \tilde{y}_{a}(t)\right.
$$

Taking into account the inequalities above and the error definition (45) we obtain

$$
\dot{V}_{4}(t) \leq-\tilde{\boldsymbol{e}}^{\top}(t) Q \tilde{\boldsymbol{e}}(t)-\tilde{y}_{a}^{2}(t),
$$


which implies that the error signals $\tilde{\boldsymbol{e}}(t), \tilde{\boldsymbol{k}}(t), \tilde{\boldsymbol{w}}_{f}(t), \tilde{\boldsymbol{w}}_{g}(t), \sigma_{p}^{*}-\hat{\sigma}_{p}(t), \tilde{\boldsymbol{\vartheta}}_{1}(t), \tilde{\boldsymbol{w}}_{a}(t), \tilde{\boldsymbol{\vartheta}}_{2}(t)$ and $\sigma_{f}^{*}-\hat{\sigma}_{f}(t)$ are bounded. Then, the estimates $\hat{\boldsymbol{k}}(t), \hat{\boldsymbol{w}}_{f}(t), \hat{\boldsymbol{w}}_{g}(t), \hat{\sigma}_{p}(t), \hat{\boldsymbol{\vartheta}}_{1}(t), \hat{\boldsymbol{w}}_{a}(t), \hat{\boldsymbol{\vartheta}}_{2}(t)$ and $\hat{\sigma}_{f}(t)$ are bounded as well. At this moment we cannot conclude anything about the boundedness of $\hat{\boldsymbol{e}}(t), \boldsymbol{e}(t), \hat{y}_{a}(t), \tilde{y}_{a}(t)$ or $y_{a}(t)$. However, we can integrate the inequality (54) and obtain

$$
\int_{0}^{t}\left[\tilde{\boldsymbol{e}}^{\top}(\tau) Q \tilde{\boldsymbol{e}}(\tau)+\tilde{y}_{a}^{2}(\tau)\right] d \tau \leq V_{4}(0)-V_{4}(t) .
$$

From Lemma 2 it follows that $V_{4}(t)$ is bounded, therefore $\tilde{\boldsymbol{e}}(t) \in \mathcal{L}_{2}$ and $\tilde{y}_{a}(t) \in \mathcal{L}_{2}$.

\section{B. Adaptive control design}

We are interested in designing a control signal $u(t)$ for the cascaded system

$$
\begin{aligned}
\dot{\hat{\boldsymbol{e}}}(t) & =A_{m} \hat{\boldsymbol{e}}(t)+\boldsymbol{b}\left[\hat{\boldsymbol{k}}^{\top}(t) \boldsymbol{x}(t)+\hat{\boldsymbol{w}}_{f}^{\top}(t) \boldsymbol{\varphi}_{f}(\boldsymbol{x})+\hat{\boldsymbol{w}}_{g}^{\top}(t) \boldsymbol{\varphi}_{g}(\boldsymbol{x}) \hat{y}_{a}(t)+\hat{\sigma}_{p}(t) \operatorname{sign}(\psi(t))-\nu y_{c}(t)\right] \\
\varepsilon^{-1} \dot{\boldsymbol{\beta}}_{2}(t) & =\left(A_{0}-\boldsymbol{b}^{0} \boldsymbol{k}_{0}^{\top}\right) \boldsymbol{\beta}_{2}(t)+\boldsymbol{b}^{0} u(t),
\end{aligned}
$$

where $\hat{y}_{a}(t)=\hat{\boldsymbol{q}}^{\top}(t) \boldsymbol{\chi}_{1}(t)+\hat{\boldsymbol{b}}_{a}(t) \boldsymbol{\beta}_{2}(t)+\hat{\boldsymbol{w}}_{a}^{\top}(t) \boldsymbol{\chi}_{a}(t)+\hat{\sigma}_{f}(t) \operatorname{sign}(\psi(t))$, such that $\hat{\boldsymbol{e}}(t) \rightarrow 0$ as $t \rightarrow \infty$. We notice that the second system in (56) is of relative degree $r$, stable and minimum phase. Therefore, it can be represented in the normal form

$$
\begin{aligned}
\varepsilon^{-1} \dot{\boldsymbol{\omega}}_{2}(t) & =A_{0} \boldsymbol{\omega}_{2}(t)+\boldsymbol{b}_{0}\left[-\boldsymbol{k}_{a}^{\top} \boldsymbol{\beta}_{2}(t)+u(t)\right] \\
\varepsilon^{-1} \dot{\boldsymbol{\xi}}_{a}^{c}(t) & =A_{0}^{c} \boldsymbol{\xi}_{a}^{c}(t)+\boldsymbol{b}_{a}^{c} \boldsymbol{\omega}_{2}(t),
\end{aligned}
$$

where the triplet $\left(\boldsymbol{c}_{0}^{\top} \in \mathbb{R}^{r}, A_{0} \in \mathbb{R}^{r \times r}, \boldsymbol{b}_{0} \in \mathbb{R}^{r}\right)$ is the normal form of representation of chain of $r$ integrators, and the eigenvalues of $A_{0}^{c} \in \mathbb{R}^{(m-r) \times(m-r)}$ are the stable zeros of the minimum phase transfer function $G(s)=\boldsymbol{c}_{0}^{\top}\left(\varepsilon s \mathbb{I}-A_{0}+\boldsymbol{b}^{0} \boldsymbol{k}_{0}^{\top}\right) \boldsymbol{b}^{0}$. The control design besides the tracking task must guarantee also the boundedness of $\boldsymbol{\omega}_{2}(t)$. Then the boundedness of $\boldsymbol{\xi}_{a}^{c}(t)$ will follow from the input-to-state stability of the internal dynamics.

To this end consider a change of variables $\boldsymbol{z}^{o}(t)=T_{o} \boldsymbol{\omega}_{2}(t)$, where $T_{o}=\operatorname{diag}\left(1, \varepsilon, \ldots, \varepsilon^{r-1}\right)$. In these new variables, the second equation in (56) takes the form

$$
\begin{aligned}
\dot{z}_{i}^{o}(t) & =z_{i+1}^{o}(t), \quad i=1, \ldots, r-1 \\
\varepsilon^{-r} \dot{z}_{r}^{o}(t) & =-\boldsymbol{k}_{0}^{\top} T_{o}^{-1} \boldsymbol{z}^{0}(t)+u(t) .
\end{aligned}
$$

We notice that

$$
\hat{\boldsymbol{w}}_{g}^{\top}(t) \boldsymbol{\varphi}_{g}(\boldsymbol{x}) \hat{y}_{a}(t)=\hat{\boldsymbol{w}}_{g}^{\top}(t) \boldsymbol{\varphi}_{g}(\boldsymbol{x})\left[\hat{\boldsymbol{q}}^{\top}(t) \boldsymbol{\chi}_{1}(t)+\hat{\omega}(t) z_{1}^{o}(t)+\hat{\boldsymbol{w}}_{a}^{\top}(t) \boldsymbol{\chi}_{3}(t)+\hat{\sigma}_{f}(t) \operatorname{sign}(\psi(t))\right],
$$

where the inequality $\hat{\omega}(t)>0$ is guaranteed by the adaptive law (48). Therefore, we design the stabilizing function $z_{1}^{o}(t)=\hat{\alpha}_{1}\left(t, \boldsymbol{x}_{p}\right)$ for the tracking error prediction model as follows

$$
\begin{aligned}
\hat{\alpha}_{1}\left(t, \boldsymbol{x}_{p}\right) & =\frac{-\hat{\boldsymbol{k}}^{\top}(t) \boldsymbol{x}_{p}(t)-\hat{\boldsymbol{w}}_{f}^{\top}(t) \boldsymbol{\varphi}_{f}\left(\boldsymbol{x}_{p}\right)-\hat{\sigma}_{p}(t) \operatorname{sign}(\psi(t))+\nu y_{c}(t)}{\hat{\vartheta}_{2 r}(t) \hat{\boldsymbol{w}}_{g}^{\top}(t) \boldsymbol{\varphi}_{g}\left(\boldsymbol{x}_{p}\right)} \\
& -\frac{\hat{\boldsymbol{q}}^{\top}(t) \boldsymbol{\chi}_{1}(t)+\hat{\boldsymbol{w}}_{a}^{\top}(t) \boldsymbol{\chi}_{3}(t)+\hat{\sigma}_{f}(t) \operatorname{sign}(\psi(t))}{\hat{\vartheta}_{2 r}(t)}
\end{aligned}
$$

If we introduce the variable $\eta_{1}(t)$ as in the previous section, that is $\eta_{1}(t)=z_{1}^{o}(t)-\hat{\alpha}_{1}\left(t, \boldsymbol{x}_{p}\right)$, the error prediction model takes the convenient form

$$
\dot{\hat{\boldsymbol{e}}}(t)=A_{m} \hat{\boldsymbol{e}}(t)+\boldsymbol{b} \hat{\boldsymbol{w}}_{g}^{\top}(t) \boldsymbol{\varphi}_{g}\left(\boldsymbol{x}_{p}\right) \eta_{1}(t) .
$$

However, the dynamics of $\eta_{1}(t)$ involves the derivative of $\hat{\alpha}_{1}\left(t, \boldsymbol{x}_{p}\right)$, which is not available in this case because of unknown terms in the plant's dynamics. One remedy for the situation is the command filtered approach 
presented in. ${ }^{3}$ Following Ref. ${ }^{3}$ we first filter the stabilizing function $\hat{\alpha}_{1}\left(t, \boldsymbol{x}_{p}\right)$ through a second order stable filter

$$
\begin{aligned}
& \dot{z}_{11}(t)=\omega_{\alpha} z_{12}(t) \\
& \dot{z}_{12}(t)=-2 \zeta_{\alpha} \omega_{\alpha} z_{12}(t)-\omega_{\alpha}\left[z_{11}(t)-\hat{\alpha}_{1}\left(t, x_{p}\right)\right]
\end{aligned}
$$

then use the error variable $\hat{\eta}_{1}(t)=z_{1}^{o}(t)-z_{11}(t)$ instead of $\eta_{1}(t)$. The tracking error prediction model now can be written as

$$
\dot{\hat{\boldsymbol{e}}}(t)=A_{m} \hat{\boldsymbol{e}}(t)+\boldsymbol{b}_{p} \hat{\boldsymbol{w}}_{g}^{\top}(t) \boldsymbol{\varphi}_{g}\left(\boldsymbol{x}_{p}\right) \hat{\eta}_{1}(t)+\boldsymbol{b}_{p} \hat{\boldsymbol{w}}_{g}^{\top}(t) \boldsymbol{\varphi}_{g}\left(\boldsymbol{x}_{p}\right)\left[z_{11}(t)-\hat{\alpha}_{1}\left(t, \boldsymbol{x}_{p}\right)\right]
$$

The compensated error $\overline{\boldsymbol{e}}(t)=\hat{\boldsymbol{e}}(t)-\hat{\boldsymbol{\xi}}(t)$, where $\hat{\boldsymbol{\xi}}(t)$ is defined according to equation

$$
\dot{\hat{\boldsymbol{\xi}}}(t)=A_{m} \hat{\boldsymbol{\xi}}(t)+\boldsymbol{b}_{p} \hat{\boldsymbol{w}}_{g}^{\top}(t) \boldsymbol{\varphi}_{g}\left(\boldsymbol{x}_{p}\right)\left[z_{11}(t)-\hat{\alpha}_{1}\left(t, \boldsymbol{x}_{p}\right)+\hat{\xi}_{a 1}(t)\right]
$$

satisfies the equation

$$
\dot{\overline{\boldsymbol{e}}}(t)=A_{m} \overline{\boldsymbol{e}}(t)+\boldsymbol{b}_{p} \hat{\boldsymbol{w}}_{g}^{\top}(t) \boldsymbol{\varphi}_{g}\left(\boldsymbol{x}_{p}\right) \bar{\eta}_{1}(t),
$$

where $\bar{\eta}_{1}(t)=\hat{\eta}_{1}(t)-\hat{\xi}_{a 1}(t), \hat{\xi}_{a 1}(t)$ is to be defined in the next step.

Following the block-backstepping ${ }^{7}$ and command filtering ${ }^{3}$ procedures, the stabilizing functions $\hat{\alpha}_{i}, i=$ $2, \ldots, r$ are defined as follows.

$$
\begin{gathered}
\hat{\alpha}_{2}\left(t, \boldsymbol{x}_{p}\right)=-\lambda_{1} \hat{\eta}_{1}(t)+\omega_{\alpha} z_{12}(t)-(\hat{\boldsymbol{e}}(t)-\hat{\boldsymbol{\xi}}(t))^{\top} P \boldsymbol{b}_{p} \hat{\boldsymbol{w}}_{g}^{\top}(t) \boldsymbol{\varphi}_{g}\left(\boldsymbol{x}_{p}\right) \\
\hat{\alpha}_{i}\left(t, \boldsymbol{x}_{p}\right)=-\lambda_{i-1} \hat{\eta}_{i-1}(t)-\hat{\eta}_{i-2}(t)+\omega_{\alpha} z_{i-1,2}(t), \quad i=3, \ldots, r,
\end{gathered}
$$

where $\hat{\eta}_{i}(t)=z_{i}^{o}(t)-z_{i 1}(t), z_{i 1}(t)$ is generated through the stable command filter

$$
\begin{aligned}
& \dot{z}_{i 1}(t)=\omega_{\alpha} z_{i 2}(t) \\
& \dot{z}_{i 2}(t)=-2 \zeta_{\alpha} \omega_{\alpha} z_{i 2}(t)-\omega_{\alpha}\left[z_{i 1}(t)-\hat{\alpha}_{i}\left(t, \boldsymbol{x}_{p}\right)\right] .
\end{aligned}
$$

The prediction models $(56)$ in terms of error signals $\hat{\eta}_{1}(t), \ldots, \hat{\eta}_{r}(t)$ can be written in the following compact form

$$
\begin{aligned}
& \underbrace{\left[\begin{array}{c}
\dot{\hat{\boldsymbol{e}}}(t) \\
\dot{\hat{\eta}}_{1}(t) \\
\vdots \\
\dot{\hat{\eta}}_{r-1}(t)
\end{array}\right]}_{\dot{\hat{\boldsymbol{x}}}(t)}=\underbrace{\left[\begin{array}{c}
A_{m} \hat{\boldsymbol{e}}(t)+\boldsymbol{b}_{p} \hat{\boldsymbol{w}}_{g}^{\top}(t) \boldsymbol{\varphi}_{g}\left(\boldsymbol{x}_{p}\right) \hat{\eta}_{1}(t)+\boldsymbol{b}_{p} \hat{\boldsymbol{w}}_{g}^{\top}(t) \boldsymbol{\varphi}_{g}\left(\boldsymbol{x}_{p}\right)\left[z_{11}(t)-\hat{\alpha}_{1}\left(t, \boldsymbol{x}_{p}\right)\right] \\
-\lambda_{1} \hat{\eta}_{1}(t)+\hat{\eta}_{2}(t)-(\hat{\boldsymbol{e}}(t)-\hat{\boldsymbol{\xi}}(t))^{\top} P \boldsymbol{b}_{p} \hat{\boldsymbol{w}}_{g}^{\top}(t) \boldsymbol{\varphi}_{g}\left(\boldsymbol{x}_{p}\right)+z_{21}(t)-\hat{\alpha}_{2}\left(t, \boldsymbol{x}_{p}\right) \\
\vdots \\
-\hat{\eta}_{r-2}(t)-\lambda_{r-1} \hat{\eta}_{r-1}(t)+z_{r 1}(t)-\hat{\alpha}_{r}\left(t, \boldsymbol{x}_{p}\right)
\end{array}\right]}_{\hat{\boldsymbol{f}}(t, \hat{\boldsymbol{x}})} \underbrace{\left[\begin{array}{c}
0 \\
0 \\
\vdots \\
1
\end{array}\right]}_{\hat{\boldsymbol{g}}} \hat{\eta}_{r}(t) \\
& \varepsilon^{-r} \dot{\hat{\eta}}_{r}(t)=-\varepsilon^{-r} \dot{z}_{r 1}(t)+u(t)-\boldsymbol{k}_{0}^{\top} T_{o}^{-1} \boldsymbol{z}^{o}(t)
\end{aligned}
$$

The compensated error is defined as $\bar{\eta}_{i}(t)=\hat{\eta}_{i}(t)-\hat{\xi}_{a i}(t)$, where $\hat{\xi}_{a i}(t)$ is generated by the dynamics

$$
\begin{aligned}
\dot{\hat{\xi}}_{a i}(t) & =-\lambda_{i} \hat{\xi}_{a i}(t)+z_{i+1,1}(t)-\hat{\alpha}_{i}\left(t, \boldsymbol{x}_{p}\right)+\hat{\xi}_{a, i+1}(t), \quad i=1, r-1 \\
\hat{\xi}_{a, r} & =0 .
\end{aligned}
$$

From the above constructions it follows that $\bar{\eta}_{r}(t)=\hat{\eta}_{r}(t)$. The dynamics of the compensated error can be written as

$$
\begin{aligned}
\dot{\bar{\eta}}_{1}(t) & =-\lambda_{1} \bar{\eta}_{1}(t)+\eta_{2}(t)-\overline{\boldsymbol{e}}^{\top}(t) P \boldsymbol{b}_{p} \hat{\boldsymbol{w}}_{g}^{\top}(t) \boldsymbol{\varphi}_{g}\left(\boldsymbol{x}_{p}\right) \\
\dot{\bar{\eta}}_{i}(t) & =-\bar{\eta}_{i-1}(t)-\lambda_{i} \bar{\eta}_{i}(t)+\bar{\eta}_{i+1}(t), \quad i=2, \ldots, r-1 \\
\varepsilon^{-r} \dot{\bar{\eta}}_{r}(t) & =-\varepsilon^{-r} \dot{z}_{r 1}(t)+u(t)-\boldsymbol{k}_{0}^{\top} T_{o}^{-1} \boldsymbol{z}^{o}(t) .
\end{aligned}
$$


We apply the singular perturbation method to the cascaded system

$$
\begin{aligned}
& {\left[\begin{array}{c}
\dot{\overline{\boldsymbol{e}}}(t) \\
\dot{\bar{\eta}}_{1}(t) \\
\vdots \\
\dot{\bar{\eta}}_{r-1}(t)
\end{array}\right] } \underbrace{\left[\begin{array}{c}
A_{m} \overline{\boldsymbol{e}}(t)+\boldsymbol{b}_{p} \hat{\boldsymbol{w}}_{g}^{\top}(t) \boldsymbol{\varphi}_{g}\left(\boldsymbol{x}_{p}\right) \bar{\eta}_{1}(t) \\
-\lambda_{1} \bar{\eta}_{1}(t)+\bar{\eta}_{2}(t)-\overline{\boldsymbol{e}}^{\top}(t) P \boldsymbol{b}_{p} \hat{\boldsymbol{w}}_{g}^{\top}(t) \boldsymbol{\varphi}_{g}\left(\boldsymbol{x}_{p}\right) \\
\vdots \\
\varepsilon^{-r} \dot{\bar{\eta}}_{r}(t)
\end{array}\right]}_{\dot{\dot{\boldsymbol{x}}(t)}}+\underbrace{\left[\begin{array}{c}
0 \\
0 \\
\vdots \\
1
\end{array}\right]}_{\overline{\boldsymbol{f}}(t, \overline{\boldsymbol{x}})} \underbrace{\left[\begin{array}{c}
0 \\
\bar{\eta}_{r-2}(t)-\lambda_{r-1} \bar{\eta}_{r-1}(t)
\end{array}\right]}_{\overline{\boldsymbol{g}}} \\
&=-\varepsilon^{-r} \dot{z}_{r 1}(t)+u(t)-\boldsymbol{k}_{0}^{\top} T_{o}^{-1} \boldsymbol{z}^{o}(t)
\end{aligned}
$$

by introducing slow time variable $\tau=\varepsilon^{r} t$. In the slow time the systems are written as

$$
\begin{aligned}
\varepsilon^{r} \frac{d \overline{\boldsymbol{x}}(\tau)}{d \tau} & =\overline{\boldsymbol{f}}(t, \overline{\boldsymbol{x}})+\overline{\boldsymbol{g}} \bar{\eta}_{r}(t) \\
\frac{d \bar{\eta}_{r}(t)}{d \tau} & =-\frac{d z_{r 1}(\tau)}{d \tau}+u(t)-\boldsymbol{k}_{0}^{\top} T_{o}^{-1} \boldsymbol{z}^{0}(t)
\end{aligned}
$$

This system is in the form of the standard singular perturbation model, if the algebraic equation

$$
\overline{\boldsymbol{f}}(t, \overline{\boldsymbol{x}})+\overline{\boldsymbol{g}} \bar{\eta}_{r}(t)=0
$$

has isolated roots. We are not concerned about finding all the roots of the equation (72), but we are interested in the root $\overline{\boldsymbol{x}}^{*}=\left[\begin{array}{lllll}\overline{\boldsymbol{e}}=0 & \bar{\eta}_{1}=0 & \ldots & \bar{\eta}_{r-2}=0 & \bar{\eta}_{r-1}=\lambda_{r-1}^{-1} \hat{\eta}_{r}(t)\end{array}\right]^{\top}$, which we denote by $\overline{\boldsymbol{x}}^{*}=\boldsymbol{h}\left(t, \bar{\eta}_{r}\right)$. Introducing a change of variables $\boldsymbol{\zeta}(\tau)=\overline{\boldsymbol{x}}(\tau)-\boldsymbol{h}\left(t, \bar{\eta}_{r}\right)$, the boundary layer system is obtained from the first equation in (71) as follows

$$
\frac{d \boldsymbol{\zeta}(t)}{d t}=\overline{\boldsymbol{f}}\left(t, \boldsymbol{\zeta}+\boldsymbol{h}\left(t, \bar{\eta}_{r}\right)+\overline{\boldsymbol{g}} \bar{\eta}_{r}(t)=\overline{\boldsymbol{f}}(t, \boldsymbol{\zeta}) .\right.
$$

The system (73) has its equilibrium at the origin. The following lemma shows that it is globally exponentially stable.

Lemma 4 The boundary layer system (73) is globally exponentially stable.

Proof. Consider a candidate Lyapunov function

$$
V_{5}(t)=\overline{\boldsymbol{e}}^{\top}(t) P \overline{\boldsymbol{e}}(t)+\bar{\eta}_{1}^{2}(t)+\cdots+\bar{\eta}_{r-1}^{2}(t)
$$

It is straightforward to compute its derivative along the solutions of system (73). After some algebra we obtain

$$
\dot{V}_{5}(t)=-\overline{\boldsymbol{e}}^{\top}(t) Q \overline{\boldsymbol{e}}(t)-\lambda_{1} \bar{\eta}_{1}^{2}(t)-\cdots-\lambda_{r-1} \bar{\eta}_{r-1}^{2}(t) .
$$

Denoting $\mu=\lambda_{\max }^{-1}(P) \min \left[\lambda_{\min }^{-1}(Q), \lambda_{1}, \ldots, \lambda_{r-1}\right]$ the following inequality can be readily derived

$$
\dot{V}_{5}(t) \leq-\mu v_{5}(t)
$$

which along with the radial unboundedness of $V_{5}(t)$ implies the global exponential stability of (73).

The reduced model is derived from the second equation in (71) by substituting $\overline{\boldsymbol{x}}(\tau)=\boldsymbol{h}\left(\tau, \bar{\eta}_{r}\right)$ and $\varepsilon=0$. Since the only term $\boldsymbol{k}_{0}^{\top} T_{o}^{-1} \boldsymbol{z}^{0}(t)$ does not explicitly depend on $\overline{\boldsymbol{x}}$, we can write the reduced system in the form

$$
\frac{d \bar{\eta}_{r}(\tau)}{d \tau}=-\frac{d z_{r 1}(\tau)}{d \tau}+v(t)-\boldsymbol{k}_{0}^{\top} T_{o}^{-1} \boldsymbol{z}^{0}(t)
$$

The control signal $v(\tau)$ is designed in the actual time scale as

$$
u(t)=-\lambda_{r} \bar{\eta}_{r}(t)+\boldsymbol{k}_{0}^{\top} T_{o}^{-1} \boldsymbol{z}^{0}(t)+\varepsilon^{-r} \omega_{\alpha} z_{r 2}(t),
$$

which in the actual time scale results in the following closed-loop reduced system

$$
\dot{\bar{\eta}}_{r}(t)=-\varepsilon^{r} \lambda_{r} \bar{\eta}_{r}(\tau)
$$


Obviously the reduced system has a globally exponentially stable equilibrium at the origin. The rest of the conditions of Theorem 1 follow from the smoothness and boundedness assumptions imposed on the reference input, as well as on the plant's and actuators's dynamics. Therefore, for all $t \geq 0$ the singular perturbation problem (71) has a unique solution $\overline{\boldsymbol{x}}(t, \varepsilon), \bar{\eta}_{r}(t, \varepsilon)$ with initial conditions $\overline{\boldsymbol{x}}(0)$ and $\left.\bar{\eta}_{r}(0)\right)$ respectively, and the relationship

$$
\bar{\eta}_{r}(t, \varepsilon)-\bar{\eta}_{r}(t)=O(\varepsilon)
$$

holds, where $\bar{\eta}_{r}(t)$ is the unique solution of the reduced system with initial condition $\bar{\eta}_{r}(0)=z_{r}^{o}(0)-z_{r 1}(0)$. Moreover, there exists a time instance $T(\epsilon)$ such that the relationship

$$
\overline{\boldsymbol{x}}(t, \varepsilon)-\boldsymbol{h}\left(t, \bar{\eta}_{r}(t)\right)=O(\varepsilon)
$$

holds for all $t \geq T(\varepsilon)$. From the exponential stability of (79) it follows that there exists time instant $T_{1}$ such that $\bar{\eta}_{r}(t)=O(\varepsilon)$ for $t \geq T_{1}$. Then, from the definition of $\boldsymbol{h}\left(t, \bar{\eta}_{r}(t)\right)$ it can be seen that the relationship $\boldsymbol{h}\left(t, \bar{\eta}_{r}(t)\right)=O(\varepsilon)$ holds for $t \geq T_{1}$. Therefore,

$$
\overline{\boldsymbol{x}}(t, \varepsilon)=O(\varepsilon)
$$

holds for $t \geq \max \left[T(\epsilon), T_{1}\right]$. Next, we apply the result of Ref. ${ }^{3}$ to the error systems (19) and (67). Since $\overline{\boldsymbol{x}}(t, \varepsilon)=O(\varepsilon)$ and $\bar{\eta}_{r}(t)=O(\varepsilon)$ for all $t \geq \max \left[T(\varepsilon), T_{1}\right]$, it can be shown that

$$
\begin{aligned}
\hat{\boldsymbol{x}}(t, \varepsilon) & =O(\varepsilon)+O\left(\omega_{\alpha}^{-1}\right) \\
\hat{\eta}_{r}(t, \varepsilon) & =O(\varepsilon)+O\left(\omega_{\alpha}^{-1}\right)
\end{aligned}
$$

for all $t \geq \max \left[T(\varepsilon), T_{1}\right]$, where $\left[\hat{\boldsymbol{x}}(t, \varepsilon) \hat{\eta}_{r}(t, \varepsilon)\right]$ is the solution of the error prediction system (67).

From (83) it follows that $\hat{\boldsymbol{e}}(t)$ and $\hat{\boldsymbol{\eta}}(t)$ are bounded. Then, from Lemma 2 it follows that $\boldsymbol{e}(t)$ is bounded. Since the reference command $y_{c}(t)$ is bounded, $\boldsymbol{x}_{m}(t)$ is bounded, implying the boundedness of $\boldsymbol{x}_{p}(t)$. Since Lemma 2 guarantees the boundedness of the parameter estimates, it follows that the stabilizing functions $\alpha_{i}\left(t, \boldsymbol{x}_{p}\right), i=1, \ldots, r$ are bounded, implying also boundedness of $\left(z_{i 1}(t), z_{i 2}(t)\right), i=1, \ldots, r$. Then from the definition of error signal $\hat{\eta}(t)$ it follows that $\boldsymbol{z}^{o}(t)$ is bounded, implying the boundedness of $\hat{y}_{a}(t)$ and $\boldsymbol{\beta}_{2}(t)$. Then the control signal $u(t)$ is bounded as well. From the the definition of $\hat{y}_{a}(t)(44)$ it follows that $\boldsymbol{\omega}_{1}(t)$ is bounded, hence $\boldsymbol{\beta}_{1}(t)$ is bounded. Then from the filter equation (42) it follows that $y_{a}(t)$ is bounded. That is, all closed loop signals are bounded. Therefore, $\dot{\tilde{\boldsymbol{e}}}(t)$ and $\dot{\tilde{y}}_{a}(t)$ are bounded. From the Barabalat's ${ }^{14}$ it follows that $\tilde{\boldsymbol{e}}(t) \rightarrow 0$ and $\tilde{y}_{a}(t) \rightarrow 0$ as $t \rightarrow \infty$.

The obtained results are formulated in the form of the following theorem.

Theorem 2 Consider the uncertain plant (8) and slow actuator (9). The adaptive controller given by (28), along with the stabilizing functions (14) and (18), auxiliary filters (61) and (66), the prediction models (40) and (44), and the adaptive laws (46), (47) and (48), guarantees that the plant's output tracks the output of the given reference model (10) with the error directly proportional to the small parameter in the actuator's dynamics and inversely proportional to the frequency of the filters (61).

\section{Simulation Example}

We demonstrate the benefits of the approach on a first order unstable system

$$
\dot{x}_{p}(t)=x_{p}^{2}(t)+x_{a}(t),
$$

controlled by the actuator

$$
\dot{x}_{a}(t)=-0.1\left(x_{a}(t)-u(t)\right) .
$$

For this simulation, the control objective is to track a reference command $y_{c}(t)=1$. That is $e(t)=x_{p}(t)-1$. The control signal $u_{b}(t)$ takes the form

$$
\begin{aligned}
\eta(t) & =x_{a}(t)+a_{m} e(t)+x_{p}^{2}(t) \\
u_{b}(t) & =-k_{a} \eta(t)+x_{a}(t)-\varepsilon^{-1} e(t)-\varepsilon^{-1}\left\{a_{m}^{2} e(t)-a_{m} \eta(t)-2 x_{p}(t)\left[x_{p}^{2}(t)+x_{a}(t)\right]\right\},
\end{aligned}
$$




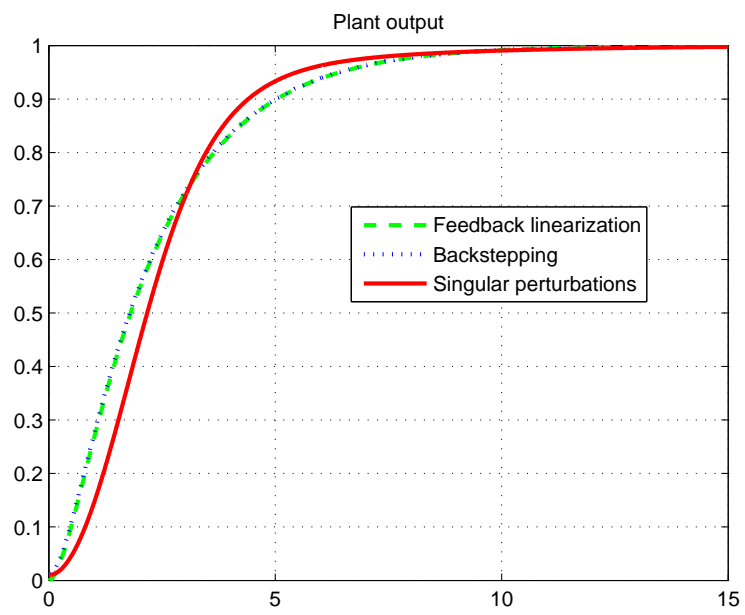

Figure 1. Output tracking performance for different control strategies.

whereas the signal $u_{p}(t)$ has form

$$
u_{p}(t)=-k_{a} \eta(t)+x_{a}(t)+\varepsilon^{-1}\left\{2\left(\frac{1}{a_{m}} \eta(t)+1\right)^{3}+x_{a}(t)\left(\frac{1}{a_{m}} \eta(t)+1\right)\right\} .
$$

Figure 1 displays the tracking performance for three controllers: feedback linearization, backstepping design and proposed design with time scale reparation. For the backstepping design and proposed design the control gains are the same: $a_{m}=3, k_{a}=1$. For the feedback linearization the control gains are chosen such that the eigenvalues of the resulting error systems are the same. As it can be seen from the plots the tracking is almost the same. The actuator's output performance is displayed in Fig. 2 and the control signals

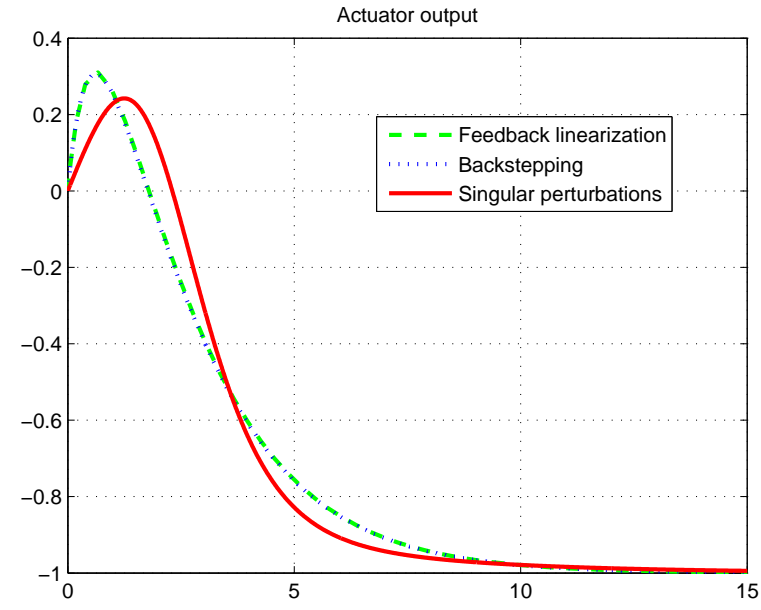

Figure 2. Actuator's output performance for different control strategies.

are displayed in Fig. 3. As it can be seen the magnitude of the control signal generated by the singular perturbations method based approach is substantially smaller the that of generated by feedback linearization and backstepping design.

We notice that with the control signal $u_{p}(t)$, the error system that is comprised of the boundary layer system and reduced system has eigenvalues -3 and -0.1 . Next we run the simulation for the feedback linearization and backstepping approach with the gains chosen such that the error system has poles at the 


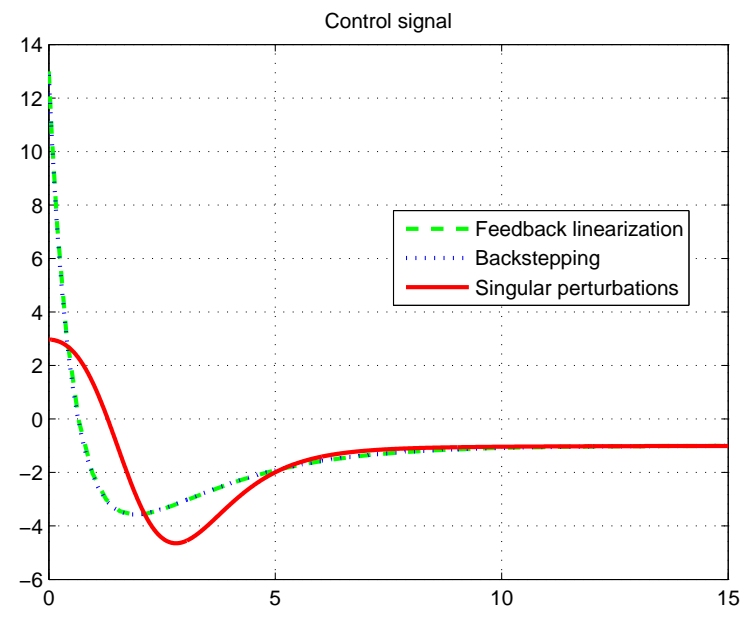

Figure 3. Control signals for different control strategies.

same location. The tracking performance and the corresponding control signals are displayed in Fig. and Fig. respectively. As it can be seen from the figures the tracking is sluggish for both designs, as it is expected, though the control signals have smaller magnitude.

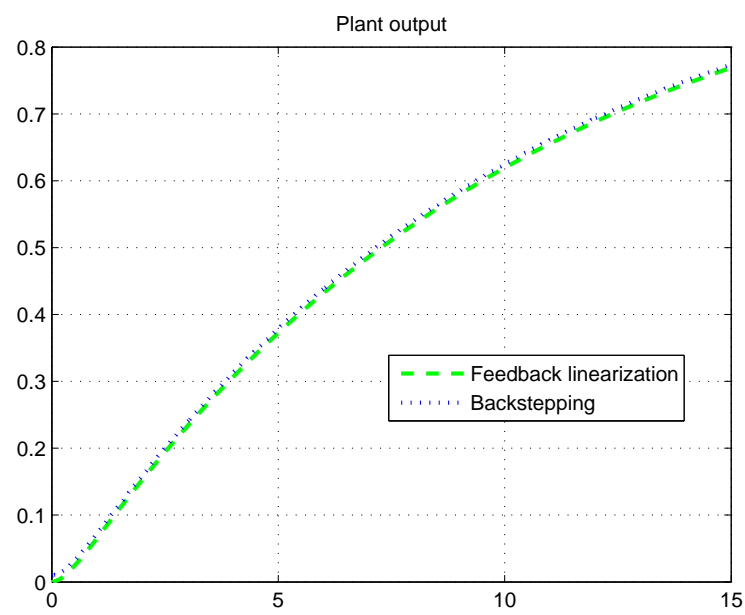

Figure 4. Output tracking performance for backstepping and feedback linearization with poles at -3 and -0.1 .

\section{Conclusion}

We considered a problem of controlling a plant with a slow actuator. It has been shown that the singular perturbations method can be applied for this problem. The resulting control signal has substantially smaller magnitude than one generated by conventional design technique, although the tracking error can be guaranteed to be only bounded with the bound proportional to the small parameter in actuator's dynamics.

\section{Acknowledgment}

The authors wish to thank Dr. Eugene Lavretsky of The Boeing Company for fruitful discussions. 


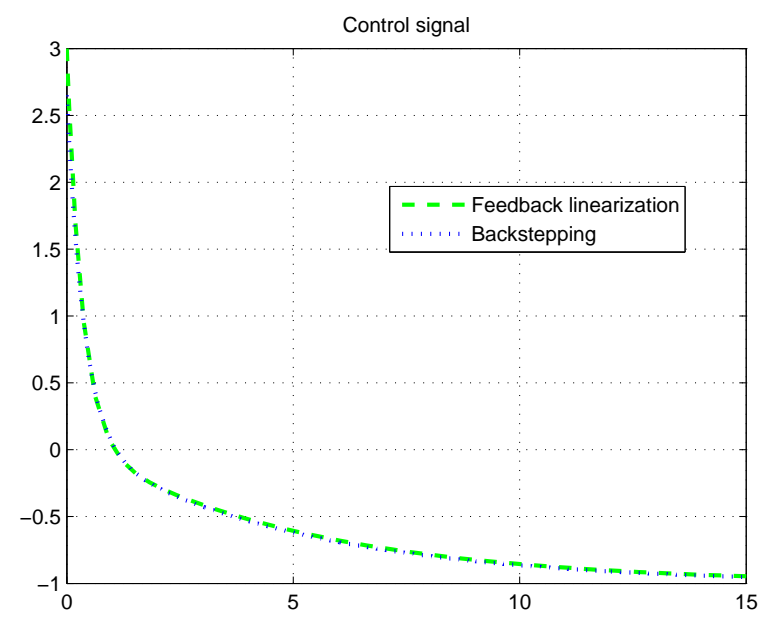

Figure 5. Control signals for backstepping and feedback linearization with poles at -3 and -0.1 .

\section{References}

${ }^{1}$ C.-C. Chen. Global Exponential Stabilization for Nonlinear Singularly Perturbed Systems. IEE Ptoc. - Control Theory Appl., 45(4):377-382, July 1998.

${ }^{2}$ P. D. Christofides and A. R. Teel. Singular Perturbations and Input-to-State Stability. IEEE Trans. Autom. Contr., 41(11):1645-1650, Nov 1996.

${ }^{3}$ J. A. Farrell, M. Polycarpou, M. Sharma, and W. Dong. Command Filtered Backstepping. IEEE Trans. Autom. Contr., 54(6):1391-1395, June 2009.

${ }^{4}$ N. Hovakimyan, E. Lavretsky, and C. Cao. Adaptive Dynamic Inversion via Time-Scale Seperation. IEEE Trans. Neural Networks, 19(10):1702-1711, 2008.

${ }^{5}$ H.K. Khalil. Nonlinear Systems, Third Edition. Prentice Hall, New Jersey, 2002.

${ }^{6}$ P. V. Kokotovic, H. K. Khalil, and L. O'Reilly. Singular Perturbations Methods in Control: Analysis and Design. Academic Press, New York, 1986.

${ }^{7}$ M. Krstic, I. Kanellakopoulos, and P. Kokotovic. Nonlinear and Adaptive Control Design. John Wiley \& Sons, New York, 1995.

${ }^{8}$ C. L. Lin and B. S. Chen. On the Design of Stabilizing controllers for Singularly Perturbed Systems. IEEE Trans. Autom. Contr., 37(11):1828-1834, 1992.

${ }^{9}$ K.S. Narendra and A.M. Annaswamy. Stable Adaptive Control. Prentice Hall, 1989.

${ }^{10} \mathrm{p}$. Sannuti and A. Saberi. Special Coordinate Basis for Multivariable Linear Systems - Finite and Infinite Zero Structure, Squaring Down and Decoupling. The International Journal of Control, 45:1655-1704, 1987.

${ }^{11}$ J. Park and I. Sandberg. Universal Approximation Using Radial Basis Function Networks. Neural Computation, 3:246257, 1991.

12 J. B. Pomet and L. Praly. Adaptive Nonlinear Regulation: Estimation from the Lyapunov Equation. IEEE Trans. Autom. Contr., 37(6):729-740, 1992.

${ }^{13}$ A. Saberi and H. Khalil. Qudratic-type Lyapunov Functions for Singularly Perturbed Systems. IEEE Trans. Autom. Contr., AC-29:542-550, 1984.

${ }^{14}$ S. S. Sastry and M. Bodson. Adaptive Control: Stability, Convergence and Robustness. Prentice Hall, 1989.

${ }^{15}$ J.J. Slotine and W. Li. Applied Nonlinear Control. Prentice Hall, New Jersey, 1991.

${ }^{16}$ A. N. Tikhonov. On the Dependence of the Solutions of Differential Equations on a Small Parameter. Mat. Sborni. N. S. 22(64) (in Russian), pages 193-204, 1948. 